\title{
Classical and Impulse Stochastic Control on the Optimization of Dividends with Residual Capital at Bankruptcy
}

\author{
Peimin Chen ${ }^{1}$ and $\mathrm{Bo} \mathrm{Li}^{2}$ \\ ${ }^{1}$ School of Economic Mathematics, Southwestern University of Finance and Economics, Chengdu, Sichuan 611130, China \\ ${ }^{2}$ School of Finance, Southwestern University of Finance and Economics, Chengdu, Sichuan 611130, China \\ Correspondence should be addressed to Peimin Chen; chenpeimin@swufe.edu.cn
}

Received 10 October 2016; Accepted 6 February 2017; Published 23 February 2017

Academic Editor: Yong Zhou

Copyright (C) 2017 Peimin Chen and Bo Li. This is an open access article distributed under the Creative Commons Attribution License, which permits unrestricted use, distribution, and reproduction in any medium, provided the original work is properly cited.

In this paper, we consider the optimization problem of dividends for the terminal bankruptcy model, in which some money would be returned to shareholders at the state of terminal bankruptcy, while accounting for the tax rate and transaction cost for dividend payout. Maximization of both expected total discounted dividends before bankruptcy and expected discounted returned money at the state of terminal bankruptcy becomes a mixed classical-impulse stochastic control problem. In order to solve this problem, we reduce it to quasi-variational inequalities with a nonzero boundary condition. We explicitly construct and verify solutions of these inequalities and present the value function together with the optimal policy.

\section{Introduction}

Corporate dividend policy has long engaged the attention of financial economists. The classical paper [1] by Miller and Modigliani provides the valuation formula for an infinite horizon firm under perfect certainty. In a world of perfect capital markets, they show that the dividend policy is irrelevant as a firm can always raise funds to meet the need for continuing operation. However, under a more realistic condition with imperfections such as the presence of financial constraints, information asymmetry, agency costs, taxes, risk exposure under uncertainty, transaction costs, and other frictions, it has been shown that there exists an optimal dividend policy (see [2-5]). Thus, determining the optimal dividend payouts becomes an important issue as it affects firm value. More recent models have focused on the issue of how to set the optimal dividend policy in a dynamic uncertain environment.

The valuation model used by Miller-Modigliani in 1961 can be extended to the situation of controllable business activities in a stochastic environment. During the recent decades, there have been increasing interests in applying diffusion models to financial decision problems, especially in (re)insurance modelling (see [6-17]). For most of these models, the liquid assets processes of the corporation contain a Brownian motion with drift and diffusion terms. The drift term corresponds to the expected profit per unit time, and the diffusion term represents risk exposure. By using diffusion models, many kinds of optimal dividend problems, such as in $[7,14-16]$, are discussed and optimal policies are presented in these papers. Particularly, in some papers (see $[14-16,18]$ ), authors discuss much more practical problems by considering a fixed transaction cost for each dividend payout. In [14], the optimal dividend problem without bankruptcy for insurance firms is considered under the assumptions of constant tax rate and fixed cost for dividend payout. In $[15,16]$, the author considers the general income process $X_{t}$ with drift term $\mu\left(X_{t}\right)$ and diffusion term $\sigma\left(X_{t}\right)$ and the case of bankruptcy. Moreover, numerical methods, such as the Runge-Kutta method, are implemented to simulate the Hamilton-Jacobi-Bellman (HJB) equation, which is a nonlinear differential equation. In [19], the author studies the dual risk model with a barrier strategy under the concept of bankruptcy, in which one has a positive probability to continue business despite temporary negative surplus. In [20], the author considers an insurance entity endowed with 
an initial capital and an income, modelled as a Brownian motion with drift and finds an explicit expression for the value function and for the optimal strategy in the first but not in the second case, where one has to switch to the viscosity ansatz. In [21], the authors suppose that a large insurance company can control its surplus process by reinsurance, paying dividends, or injecting capitals and obtain the explicit solutions for value function and optimal strategy.

In these papers, the value function is typically assumed to be zero when there is a bankruptcy. But in the real world, some shareholders, especially for preferred shareholders, can get some money back when a terminal bankruptcy occurs. That means for this case the value function is not zero at bankruptcy. Thus, it is very useful and necessary for us to consider this kind of problem. In this paper, we postulate that the amount of money, shareholders can obtain for the terminal bankruptcy, is a positive constant, $a$. Moreover, we assume that the liquid assets $X_{t}$ follows a process with constant drift and diffusion coefficients.

In the model of this paper, as that in [14], the dividend distribution policy is given by a purely discontinuous increasing functional. The net amount of money received by shareholders is $k \xi_{i}-K$ for the $i$-th dividends, where $\xi_{i}$ is the amount of the dividend payments, $1-k$ is the tax rate the shareholder pays, and $K$ is the fixed cost whenever the dividends are paid out. Further, $\tau_{i}$ represents the moments of dividend payments and $\lambda$ is the discount rate. Based on these assumptions, we transform the value function $V(x)$ into quasi-variational inequalities (QVI) and list out a candidate solution $v(x)$ to QVI with a positive boundary condition $v(0)=a$. Subsequently, we show that the value function can be given by $v(x)$ and the optimal policy can be presented based on the solution $v(x)$. A natural question is how to point out whether there is a bankruptcy or not in order to obtain the optimal policy under some conditions. To answer this question, some criteria are provided.

A major difficulty in this paper is that the structure of the candidate solution is uncertain since the existing interval of it has unfixed endpoints, which depends on some unknown parameters. This phenomenon does not appear in [14] and other related papers. Enlightened by the derivatives of candidate solutions, we construct the integral $I(C)$ and then discuss it by several cases for $\mu, \sigma, k, K$, and $a$. For the model mentioned above, which is restricted to stay at the bankruptcy state, it is denoted by terminal bankruptcy model as in [22].

The structure of this paper is as follows. In the next section, we provide a rigorous mathematical model for the optimal dividend problem. Then the stochastic control problem is transformed to a QVI. Moreover, some definitions and an important verification are presented. Following this, the detailed structure of candidate solutions is given under different situations in Section 3. In Section 4, the uniqueness of some unfixed parameters is verified; some formulas to calculate these parameters are proposed and some numerical examples are shown to support our theoretical results. In Section 5, we demonstrate that the candidate solutions satisfy QVI and obtain the optimal dividend policy. In the last section, we summarize our results and suggest a direction for future research.

\section{The Mathematical Model}

The proposed model considers the dividend optimization problem for a firm which can control its business activities that affect its risk and potential profit. It extends the classical Miller-Modigliani model of firm valuation to the situation of controllable business activities in a stochastic environment with a possibility of bankruptcy and a positive residual value to shareholders upon bankruptcy. The model is quite general and can be applied to any firm in which management has control on the dividend stream as well as the risk exposure. Without loss of generality, the model is cast in the framework of a large insurance company, as it possesses many nice features to best illustrate the model.

2.1. Value Function. Let $(\Omega, \mathscr{F}, P)$ be a probability space with a filtration $\left\{\mathscr{F}_{t}\right\}_{t \geq 0}$ and $W=\left\{W_{t} ; t \geq 0\right\}$ be a standard Brownian motion adapted to that filtration. Moreover, the reserve process $X=\{X(t) ; t \geq 0\}$ is a state variable, which denotes the liquid assets of the company. For an insurance company, in order to reduce risk, the risk control takes up the form of proportional reinsurance, which mathematically corresponds to decreasing the drift and diffusion coefficient by multiplying both quantities by the same factor $u(t) \epsilon$ $[0,1]$. The time of dividends is described by a sequence of increasing stopping times $\left\{\tau_{i} ; i=1,2, \ldots\right\}$ and the amounts of the dividends paid out to the shareholders, associated with the times, are represented by a sequence of random variables $\left\{\xi_{i} ; i=1,2, \ldots\right\}$. Then the controlled state process $X(t)$ before bankruptcy is given by

$$
X(t)=x+\int_{0}^{t} \mu u(s) d s+\int_{0}^{t} \sigma u(s) d W_{s}-\sum_{n=1}^{\infty} I_{\left(\tau_{n}<t\right)} \xi_{n}
$$

where $x \geq 0$ is the initial reserve and $I_{\left(\tau_{n}<t\right)}$ is an indicator function.

Let the time of bankruptcy be given by

$$
\tau=\inf \{t \geq 0: X(t)=0\} .
$$

Definition 1. Let $u: \Omega \times[0, \infty) \rightarrow[0,1]$ be an $\left\{\mathscr{F}_{t}\right\}_{t \geq 0^{-}}$ adapted process; let $\tau_{i}, i=1,2, \ldots$, be a stopping time with respect to $\left\{\mathscr{F}_{t}\right\}_{t \geq 0}$, and let the random variable $\xi_{i}, i=1,2, \ldots$, be $\left\{\mathscr{F}_{\tau_{i}}\right\}$ measurable with $0 \leq \xi_{i} \leq X\left(\tau_{i}-\right)$; then

$$
\begin{aligned}
\pi & =(u, \mathscr{T}, \xi, \tau) \\
& =\left(u ; \tau_{1}, \tau_{2}, \ldots, \tau_{n}, \ldots ; \xi_{1}, \xi_{2}, \ldots, \xi_{n}, \ldots ; \tau\right)
\end{aligned}
$$

is called an admissible control or an admissible policy. The class of all admissible controls is denoted by $\mathscr{A}(x)$.

In addition, we denote the net amount of money that shareholders receive by a function $g:[0, \infty) \rightarrow(-\infty, \infty)$ as

$$
g(\omega)=k \omega-K
$$


where the constant $K>0$ is a fixed setup cost incurred each time that a dividend is paid out, and the constant $1-k \in(0,1)$ is the tax rate at which the dividends are taxed, and $\omega$ is a real value variable with respect to the amount of liquid assets withdrawn.

A performance functional $J$ with each admissible control $\pi$ is defined by

$$
J(x, \pi)=\mathbb{E}_{x}\left[\sum_{n=1}^{\infty} e^{-\lambda \tau_{n}} g\left(\xi_{n}\right) I_{\left(\tau_{n}<\tau\right)}+e^{-\lambda \tau} a\right],
$$

which represents the total expected discounted value received by shareholders until the time of bankruptcy, where $a$ is the known amount paid out to shareholders when the terminal bankruptcy happens.

Define the value function $V(x)$ by

$$
V(x)=\sup _{\pi \in A(x)} \mathbb{E}_{x}\left[\sum_{n=1}^{\infty} e^{-\lambda \tau_{n}} g\left(\xi_{n}\right) I_{\left(\tau_{n}<\tau\right)}+e^{-\lambda \tau} a\right] .
$$

Then the optimal control $\pi^{*}=\left(u^{*}, \mathscr{T}^{*}, \xi^{*}, \tau^{*}\right)$ is a policy for which the following equality can be satisfied

$$
V(x)=J\left(x, \pi^{*}\right) .
$$

2.2. Properties of the Value Function. In this section, the QVI associated with the stochastic control problem is provided. Moreover, we derive some properties of the value function.

Proposition 2. For every $x \in[0, \infty)$, the value function $V(x)$ in (6) satisfies

$$
V(x) \leq k\left(x-e^{-\lambda \tau} a+\frac{|\mu|}{\lambda}\right) .
$$

Proof. By the same method as in [14] and letting $X(\tau)=a$ instead of $X(\tau)=0$, then the result can be obtained.

Let $g$ be given by (4); then, for a function $\phi:[0, \infty) \rightarrow \mathbb{R}$, define the maximum utility operator $M$ of it by

$$
M \phi(x)=\sup _{\omega}\{\phi(x-\omega)+g(\omega): 0<\omega \leq x\} .
$$

Suppose that the payment of dividends occurs at time 0 and the amount of it equals $\omega$; then the reserve decreases from initial position $x$ to $x-\omega$. After that, if the optimal policy is followed, the total expected utility is $k \omega-K+V(x-$ $\omega)$. Consequently, under such a policy, the total maximal expected utility would be equal to $M V(x)$. On the other hand, for each initial position $x$, suppose that there exists an optimal policy, which is optimal for the whole domain. Then the expected utility associated with this optimal policy is $V(x)$, which is greater or equal to any expected utility associated with another different policy. So, we have

$$
V(x) \geq M V(x) \text {. }
$$

Now, define

$$
\mathscr{L}^{u} v(x)=\frac{1}{2} \sigma^{2} u^{2} v^{\prime \prime}(x)+\mu u v^{\prime}(x)-\lambda v(x) .
$$

By the dynamic programming principle, we know that in the continuation region, $V(x)$ satisfies

$$
\max _{u \in[0,1]} \mathscr{L}^{u} V(x)=0
$$

The arguments in (10) and (12) give us an intuition for the following two definitions and one theorem.

Definition 3. Assume that function $v(x):[0, \infty) \rightarrow[0, \infty)$. For every $x \in[0, \infty)$ and $u \in[0,1]$, if we have

$$
\begin{aligned}
(\mathrm{QVI}) \quad & v(x) \geq M v(x), \\
& \mathscr{L}^{u} v(x) \leq 0, \\
& (v(x)-M v(x))\left(\max _{u \in[0,1]} \mathscr{L}^{u} v(x)\right)=0, \\
& v(0)=a,
\end{aligned}
$$

then we claim that $v(x)$ satisfies the quasi-variational inequalities of the control problem.

Definition 4. The control $\pi^{v}=\left(u^{v}, \mathscr{T}^{v}, \xi^{v}, \tau^{v}\right)$ is called the QVI control associated with $v$ if

$$
\begin{aligned}
& P\left\{u^{v}(t) \neq \underset{u \in[0,1]}{\arg \max } \mathscr{L}^{u} v\left(X_{t}^{v}\right), X_{t}^{v} \in \mathscr{C}\right\}=0 \\
& \tau_{0}^{v}=0 \text {, } \\
& \xi_{0}^{v}=0 \text {; } \\
& \tau_{1}^{v}=\inf \left\{t \geq 0: v\left(X^{v}(t)\right)=M v\left(X^{v}(t)\right)\right\}, \\
& \xi_{1}^{v}=\arg \sup _{0<\eta \leq X^{v}\left(\tau_{1}^{v}\right)}\left\{v\left(X^{v}\left(\tau_{1}^{v}\right)-\eta\right)+g(\eta)\right\}
\end{aligned}
$$

and, for every $n \geq 2$,

$$
\begin{aligned}
& \tau_{n}^{v}=\inf \left\{t \geq \tau_{n-1}: v\left(X^{v}(t)\right)=M v\left(X^{v}(t)\right)\right\}, \\
& \xi_{n}^{v}=\arg \sup _{0<\eta \leq X^{v}\left(\tau_{n}^{v}\right)}\left\{v\left(X^{v}\left(\tau_{n}^{v}\right)-\eta\right)+g(\eta)\right\} ; \\
& \tau^{v}=\inf \left\{t \geq 0: X^{v}(t)=0\right\} .
\end{aligned}
$$

As in Cadenillas et al. [14], we also have the following theorem.

Theorem 5. Let $v \in C^{1}([0, \infty))$ be a solution of QVI. Suppose there exists $U>0$ such that $v$ is twice continuously differentiable on $[0, U)$ and $v$ is linear on $[U, \infty)$. Then, for any $x \in[0, \infty)$,

$$
V(x) \leq v(x)
$$

Furthermore, if the QVI control $\left(u^{v}, \mathscr{T}^{v}, \xi^{v}, \tau^{v}\right)$ associated with $v$ is admissible, then $v$ coincides with the value function and the QVI control associated with $v$ is the optimal policy; that is,

$$
V(x)=v(x)=J\left(x ; u^{v}, \mathscr{T}^{v}, \xi^{v}, \tau^{v}\right) .
$$


Proof. The idea of this proof is very similar to that of Theorem 3.4 in Cadenillas et al. [14]. So, we do not show it in this paper.

\section{Smooth Solutions to the QVI Properties}

In this section, we first recall the zero boundary (no recovery) problem in Cadenillas et al. [14], and then by the similar method of this solved problem, we obtain the smooth solutions of QVI properties.

3.1. Solution for the Problem with Zero Boundary Condition. Let us consider the similar problem of QVI as follows:

$$
\begin{aligned}
\left(\mathrm{QVI}_{0}\right) \quad & v_{0}(x) \geq M v_{0}(x), \\
& \mathscr{L}^{u} v_{0}(x) \leq 0, \\
& \left(v_{0}(x)-M v_{0}(x)\right)\left(\max _{u \in[0,1]} \mathscr{L}^{u} v_{0}(x)\right)=0, \\
& v_{0}(0)=0 .
\end{aligned}
$$

Let

$$
\gamma=\frac{\lambda}{\lambda+\mu^{2} / 2 \sigma^{2}}
$$

and define

$$
\begin{aligned}
& X_{0}=\frac{(1-\gamma) \sigma^{2}}{\mu}, \\
& X_{1}=\inf \left\{x \geq 0: v_{0}(x)=M v_{0}(x)\right\}
\end{aligned}
$$

Then from Cadenillas et al. [14], we can obtain the structure of the solution of $\mathrm{QVI}_{0}$ :

$$
\begin{aligned}
& v_{0}(x) \\
& \quad= \begin{cases}C_{0} x^{\gamma}, & x \in\left[0, X_{0}\right), \\
C_{0} a_{1} e^{\theta_{+}\left(x-X_{0}\right)}+C_{0} a_{2} e^{\theta_{-}\left(x-X_{0}\right)}, & x \in\left[X_{0}, X_{1}\right), \\
v_{0}(\widetilde{X})+k(x-\widetilde{X})-K, & x \in\left[X_{1}, \infty\right),\end{cases}
\end{aligned}
$$

where $C_{0}$ is a free constant and $\theta_{+}, \theta_{-}, a_{1}$, and $a_{2}$ are given by

$$
\begin{aligned}
& \theta_{+}=\frac{-\mu+\sqrt{\mu^{2}+2 \lambda \sigma^{2}}}{\sigma^{2}}, \\
& \theta_{-}=\frac{-\mu-\sqrt{\mu^{2}+2 \lambda \sigma^{2}}}{\sigma^{2}}, \\
& a_{1}=\frac{\gamma X_{0}^{\gamma-1}-\theta_{-} X_{0}^{\gamma}}{\theta_{+}-\theta_{-}}, \\
& a_{2}=\frac{\theta_{+} X_{0}^{\gamma}-\gamma X_{0}^{\gamma-1}}{\theta_{+}-\theta_{-}},
\end{aligned}
$$

and $\widetilde{X}$ is the unique solution of the following equation:

$$
v_{0}^{\prime}(x)=k, \quad x \in\left(0, X_{1}\right) .
$$

In Cadenillas et al. [14], it has been shown that $a_{1}>0$ and $a_{2}<0$. Define a function $H(x)$ by

$$
\begin{aligned}
H(x) & \\
& = \begin{cases}\gamma x^{\gamma-1}, & x \in\left[0, X_{0}\right), \\
a_{1} \theta_{+} e^{\theta_{+}\left(x-X_{0}\right)}+a_{2} \theta_{-} e^{\theta_{-}\left(x-X_{0}\right)}, & x \in\left[X_{0}, \infty\right) .\end{cases}
\end{aligned}
$$

Let

$$
I_{0}\left(\widetilde{C}_{0}\right)=\int_{\widetilde{X}_{\widetilde{C}_{0}}^{X_{1}^{\widetilde{C}_{0}}}}^{\widetilde{C}_{0}}\left(k-\widetilde{C}_{0} H(x)\right) d x
$$

then $I_{0}\left(\widetilde{C}_{0}\right)$ is a decreasing function of $\widetilde{C}_{0}$ with the range $[0,+\infty)$, and there exists unique $\widetilde{C}_{0}, \widetilde{X}^{\widetilde{C}_{0}}$, and $X_{1}^{\widetilde{C}_{0}}$, such that $I_{0}\left(\widetilde{C}_{0}\right)=K$.

Further, Cadenillas et al. [14] show that the solution of $\mathrm{QVI}_{0}$ can be given by (21) with $C_{0}=\widetilde{C}_{0}, \widetilde{X}=\widetilde{X}^{\widetilde{C}_{0}}$, and $X_{1}=$ $X_{1}^{\widetilde{C}_{0}}$.

3.2. Smooth Solutions of QVI Properties on $\left[0, x_{1}\right]$. The difference between QVI and $\mathrm{QVI}_{0}$ is just the boundary condition. Thus, we conjecture that solutions of them may have some similarities.

3.2.1. Smooth Solutions of QVI Properties on $\left[0, x_{1}\right)$. First, as in (20), we define

$$
x_{1}=\inf \{x \geq 0: v(x)=M v(x)\} .
$$

Then, on the interval $\left(0, x_{1}\right)$, from QVI we have that

$$
\max _{u \in[0,1]} \mathscr{L}^{u} v(x)=0, \quad 0<x<x_{1} .
$$

Let $u(x) \in \mathbb{R}$ be the maximizer of the expression on the left-hand side of (28); then

$$
u(x)=-\frac{\mu v^{\prime}(x)}{\sigma^{2} v^{\prime \prime}(x)} .
$$

Putting (29) into (28), we have

$$
-\frac{\mu^{2}\left[v^{\prime}(x)\right]^{2}}{2 \sigma^{2} v^{\prime \prime}(x)}-\lambda v(x)=0 .
$$

A general solution of (30) under the boundary condition $v(0)=a$ is

$$
v(x)=C\left[\left(\frac{a}{C}\right)^{1 / \gamma}+x\right]^{\gamma},
$$

where $C$ is a free constant and $\gamma$ is presented by (19). From (29) and (31), it follows that

$$
u(x)=\frac{\mu}{(1-\gamma) \sigma^{2}}\left[x+\left(\frac{a}{C}\right)^{1 / \gamma}\right] .
$$

Since $u(x)$ above is an increasing linear function, $u(x) \leq 1$ if and only if $x \leq x_{0}$, where

$$
x_{0}=\frac{(1-\gamma) \sigma^{2}}{\mu}-\left(\frac{a}{C}\right)^{1 / \gamma}=X_{0}-\left(\frac{a}{C}\right)^{1 / \gamma} .
$$


Thus, if $x_{0}>0$ and $x_{0}<x<x_{1}$, it follows that $u(x) \geq 1$. But since the range of $u(x)$ is $[0,1]$, then we must have $u(x)=1$ for $x \in\left(x_{0}, x_{1}\right)$; consequently (28) becomes

$$
\frac{1}{2} \sigma^{2} v^{\prime \prime}(x)+\mu v^{\prime}(x)-\lambda v(x)=0, \quad x \in\left(x_{0}, x_{1}\right) .
$$

One general solution to (34) can be written by

$$
v(x)=C_{1} e^{\theta_{+}\left(x-x_{0}\right)}+C_{2} e^{\theta_{-}\left(x-x_{0}\right)}, \quad x \in\left(x_{0}, x_{1}\right),
$$

where $C_{1}$ and $C_{2}$ are free constants and $\theta_{+}$and $\theta_{-}$are given by (22). Continuity of the function $v(x)$ and its derivative $v^{\prime}(x)$ at the point $x_{0}$ implies that $C_{1}=C a_{1}$ and $C_{2}=C a_{2}$, where $C$ is a free constant and $a_{1}$ and $a_{2}$ are defined by (23).

On the other hand, if $x_{0} \leq 0$, then $u(x)=1$, for any $x \in$ $\left[0, x_{1}\right)$, and (28) becomes

$$
\begin{aligned}
\frac{1}{2} \sigma^{2} v^{\prime \prime}(x)+\mu v^{\prime}(x)-\lambda v(x) & =0, \quad x \in\left[0, x_{1}\right), \\
v(0) & =a .
\end{aligned}
$$

Solving (36), we can obtain the general solution of (36) as follows:

$$
v(x)=C_{1} e^{\theta_{+} x}+C_{2} e^{\theta_{-} x}, \quad x \in\left[0, x_{1}\right),
$$

with $C_{1}+C_{2}=a$.

Now, let us summarize the possible structure for the solution of $(28)$ on $\left[0, x_{1}\right)$. If $x_{0}>0$, then

$$
v(x)= \begin{cases}C\left[\left(\frac{a}{C}\right)^{1 / \gamma}+x\right]^{\gamma}, & x \in\left[0, x_{0}\right), \\ C a_{1} e^{\theta_{+}\left(x-x_{0}\right)}+C a_{2} e^{\theta_{-}\left(x-x_{0}\right)}, & x \in\left[x_{0}, x_{1}\right),\end{cases}
$$

where $C$ is a free constant. If $x_{0} \leq 0$, then the structure of $v(x)$ on $\left[0, x_{1}\right)$ is given by $(37)$.

Remark 6. From (33), we notice that $x_{0}$ depends on the uncertain parameter $C$, which will be estimated later. For different $C$, the sign of $x_{0}$ may be different. Moreover, it is easy to show that the assumption, $x_{0}>0$, is equivalent to $C>a X_{0}^{-\gamma}$ and $x_{0} \rightarrow 0_{+}$is equivalent to $C \rightarrow\left(a X_{0}^{-\gamma}\right)_{+}$. In addition, for (37) and (38), they are consistent at $x_{0}=0$. That is, for any $x \in\left(0, x_{1}\right)$,

$$
\begin{aligned}
& \lim _{C \rightarrow\left(a X_{0}^{-\gamma}\right)_{+}}\left(C a_{1} e^{\theta_{+}\left(x-x_{0}\right)}+C a_{2} e^{\theta_{-}\left(x-x_{0}\right)}\right) \\
& =a X_{0}^{-\gamma} a_{1} e^{\theta_{+} x}+a X_{0}^{-\gamma} a_{2} e^{\theta_{-} x} \\
& =\lim _{C_{1} \rightarrow\left(a X_{0}^{-\gamma} a_{1}\right)_{-}}\left(C_{1} e^{\theta_{+} x}+\left(a-C_{1}\right) e^{\theta_{-} x}\right) .
\end{aligned}
$$

At $C=a X_{0}^{-\gamma}$, from the consistency of two solutions, we have that $C_{1}=C a_{1}$. In addition, the condition of existence for (37) implies $C \leq a X_{0}^{-\gamma}$. Moreover, there is no conflict to denote $C_{1}$ by $C a_{1}$ when $C \leq a X_{0}^{-\gamma}$. So, we let $C_{1}=C a_{1}$ for $C \leq a X_{0}^{-\gamma}$.

Remark 7. For $v(x)$ in (38), from $a_{1}>0$ and $a_{2}<0$, it is easy to show that $v^{\prime \prime \prime}(x)>0$ on $\left[0, x_{1}\right)$, which implies that $v^{\prime}(x)$ obtained from (38) have convexity on $\left[0, x_{1}\right)$.
3.2.2. Smooth Solution of QVI Properties at $x_{1}$. From the definition of $x_{1}$, we have that $v\left(x_{1}\right)=M v\left(x_{1}\right)$. Then, by

$$
\lim _{\eta \rightarrow 0}\left(v\left(x_{1}-\eta\right)+k \eta-K\right)=v\left(x_{1}\right)-K<v\left(x_{1}\right),
$$

it follows that the maximizing sequence $\eta$ for $v\left(x_{1}\right)=M v\left(x_{1}\right)$ cannot have zero as a limiting point. So, at $x_{1}$, the supremum on the right-hand side of (9) can be taken over $\eta \in\left[\epsilon, x_{1}\right]$ for some $\epsilon>0$. Therefore, there exists $\eta\left(x_{1}\right) \in\left(0, x_{1}\right]$, such that

$$
v\left(x_{1}\right)=v\left(x_{1}-\eta\left(x_{1}\right)\right)+k \eta\left(x_{1}\right)-K .
$$

Let $\tilde{x}=x_{1}-\eta\left(x_{1}\right)$; then $0 \leq \tilde{x}<x_{1}$ and

$$
v\left(x_{1}\right)=v(\tilde{x})+k\left(x_{1}-\tilde{x}\right)-K .
$$

From (42), it follows that

$$
v\left(x_{1}\right)-v(\tilde{x})=\int_{\tilde{x}}^{x_{1}} v^{\prime}(x) d x=k\left(x_{1}-\tilde{x}\right)-K ;
$$

then we have

$$
\int_{\tilde{x}}^{x_{1}}\left(k-v^{\prime}(x)\right) d x=K .
$$

Remark 8. If $\tilde{x}=0$, then $v\left(x_{1}\right)=v(0)+k x_{1}-K=a+$ $k x_{1}-K$, which means the dividend happens once and then a bankruptcy follows. This is an important phenomenon that deserves to be discussed.

\section{Uniqueness for the Unfixed Parameters}

In Section 3, some parameters, such as $C, C_{1}$, and $C_{2}$, are unfixed numbers. In this section, we discuss the uniqueness of their corresponding parameters by two useful integral functions, whose integrands can be used to obtain solutions of QVI. As in Remark 6, we claim that $C a_{1}$ can be used to denote $C_{1}$ for $C \leq a X_{0}^{-\gamma}$.

4.1. Definitions and Properties of Two Integral Functions. From Remark 6, it is known that different $C$ will lead to two possible cases for $x_{0}$. The first case is $x_{0}>0$ and the second is $x_{0} \leq 0$. In the following, we discuss these two cases by two constructed integral functions, respectively.

Case $1\left(x_{0}>0\right)$. Let $H^{C}(x)$ be a function, with constant $C$, constructed by

$$
\begin{aligned}
& H^{C}(x) \\
& \quad= \begin{cases}\gamma\left[\left(\frac{a}{C}\right)^{1 / \gamma}+x\right]^{\gamma-1}, & x \in\left[0, x_{0}\right), \\
a_{1} \theta_{+} e^{\theta_{+}\left(x-x_{0}\right)}+a_{2} \theta_{-} e^{\theta_{-}\left(x-x_{0}\right)}, & x \in\left[x_{0}, \infty\right),\end{cases}
\end{aligned}
$$

where $x_{0}$ is also defined by (33) with $C>a X_{0}^{-\gamma}$.

Define

$$
I_{1}(C)=\int_{\widetilde{x}^{C} \vee 0}^{x_{1}^{C}}\left(k-C H^{C}(x)\right) d x,
$$


where $x_{1}^{C}$ and $\widetilde{x}^{C}$ are two nonnegative roots of the equation $k-C H^{C}(x)=0$ with $\widetilde{x}^{C}<x_{1}^{C}$ and $\widetilde{x}^{C} \vee 0$ denotes $\max \left\{\widetilde{x}^{C}, 0\right\}$. If $\widetilde{x}^{C}$ does not exist on $[0, \infty)$, then let $\widetilde{x}^{C} \vee 0=0$.

From the definitions of $H^{C}(x)$ and $I_{1}(C)$, obviously $C H^{C}(x)$ is a continuous function of $C$. Further, $\widetilde{x}^{\mathrm{C}} \vee 0$ and $x_{1}^{\mathrm{C}}$ are also continuous functions of $C$ if the existence condition of them is satisfied. So, $I_{1}(C)$ is a continuous function.

Proposition 9. Let $H^{C}(x)$ be defined by (45); then it has convexity on $[0, \infty)$ by $\left(H^{C}\right)^{\prime \prime}(x)>0$. Moreover, $\mathrm{CH}^{\mathrm{C}}(x)$ is an increasing function with respect to $\mathrm{C}$ by $\left(\mathrm{CH}^{\mathrm{C}}(x)\right)^{\prime}>$ 0 . For $I_{1}(C)$, if the condition of its existence is satisfied on some subintervals of $\left(a X_{0}^{-\gamma}, \infty\right)$, then it is a strictly decreasing function on these subintervals.

Case $2\left(x_{0} \leq 0\right)$. Let

$$
H^{C_{1}}(x)=C_{1} \theta_{+} e^{\theta_{+} x}+\left(a-C_{1}\right) \theta_{-} e^{\theta_{-} x}, \quad x \in[0, \infty),
$$

where $0<C_{1} \leq a X_{0}^{-\gamma} a_{1}$.

Define

$$
I_{2}\left(C_{1}\right)=\int_{\tilde{x}^{C_{1} \vee 0}}^{x_{1}^{C_{1}}}\left(k-H^{C_{1}}(x)\right) d x
$$

where $x_{1}^{C_{1}}$ and $\widetilde{x}^{C_{1}}$ are two nonnegative roots of the equation $k-H^{C_{1}}(x)=0$ with $\widetilde{x}^{C_{1}}<x_{1}^{C_{1}}$. If $\widetilde{x}^{C_{1}}$ does not exist on $[0, \infty)$, then let $\widetilde{x}^{C_{1}} \vee 0=0$. gives

For (48), taking the derivative of $I_{2}\left(C_{1}\right)$ with respect to $C_{1}$

$$
I_{2}^{\prime}\left(C_{1}\right)=\int_{\tilde{x}^{C_{1} \vee 0}}^{x_{1}^{C_{1}}}\left(-\theta_{+} e^{\theta_{+} x}+\theta_{-} e^{\theta_{-} x}\right) d x .
$$

Consequently, for any positive $C_{1}, I_{2}^{\prime}\left(C_{1}\right)<0$ due to the fact that $\theta_{+}>0$ and $\theta_{-}<0$. So, we have the following result.

Proposition 10. For $I_{2}\left(C_{1}\right)$, if its existence condition is satisfied on some subintervals of $\left(0, a X_{0}^{-\gamma} a_{1}\right)$, then it is a continuous and strictly decreasing function on these subintervals. Moreover, we can show that $\lim _{C_{1} \rightarrow 0} x_{1}^{C_{1}}=\infty$ and $\lim _{C_{1} \rightarrow 0} I_{2}\left(C_{1}\right)=$ $\infty$.

Remark 11. For small enough and positive $C_{1}$, it is possible that $a-C_{1}>0$. Under this situation, $H^{C_{1}}(x)$ may have no convexity since $\left(H^{C_{1}}\right)^{\prime \prime}(x)>0$ may not be satisfied.

In the following, we discuss the property of two integral functions at $x_{0}=0$.

At $x_{0}=0$ there are some common properties of $I_{1}(C)$ and $I_{2}\left(C a_{1}\right)$. Firstly, it follows that $C_{1}=C a_{1}$ and $C=a X_{0}^{-\gamma}$. Then from

$$
\begin{aligned}
a-C_{1} & =a-a X_{0}^{-\gamma} a_{1}=a\left(1-X_{0}^{-\gamma} a_{1}\right) \\
& =a X_{0}^{-\gamma}\left(X_{0}^{\gamma}-a_{1}\right)=a X_{0}^{-\gamma} a_{2},
\end{aligned}
$$

we obtain that at $x_{0}=0$ the integrands of $I_{1}(C)$ and $I_{2}\left(C_{1}\right)$ are the same. Consequently, we can conclude that if $x_{1}^{C_{1}}$ exists at $C_{1}=a X_{0}^{-\gamma} a_{1}$, then $I_{2}\left(a X_{0}^{-\gamma} a_{1}\right)=I_{1}\left(a X_{0}^{-\gamma}\right)$. To judge whether $I_{2}\left(a X_{0}^{-\gamma} a_{1}\right)>0$, we have the following result.

Proposition 12. One equivalent condition of $I_{2}\left(a X_{0}^{-\gamma} a_{1}\right)>0$ is $k>a M^{*}$, where $M^{*}$ is given by

$$
M^{*}=\left(-\frac{\theta_{-}}{\theta_{+}}\right)^{\theta_{-} /\left(\theta_{+}-\theta_{-}\right)} \cdot\left(\frac{2 \lambda}{\mu}-\theta_{-}\right) .
$$

Proof. Let $B(x)=a X_{0}^{-\gamma} a_{1} \theta_{+} e^{\theta_{+} x}+a X_{0}^{-\gamma} a_{2} \theta_{-} e^{\theta_{-} x}$. Notice that $B^{\prime \prime}(x)>0$, and so $B(x)$ has convexity. One equivalent condition of $I_{2}\left(a X_{0}^{-\gamma} a_{1}\right)>0$ is that

$$
\min _{x \in[0, \infty)} B(x)<k
$$

Solving $B^{\prime}(x)=0$, we have that

$$
x=\frac{1}{\theta_{+}-\theta_{-}} \ln \left(\frac{-a_{2} \theta_{-}^{2}}{a_{1} \theta_{+}^{2}}\right) .
$$

It can check that $\left(-a_{2} \theta_{-}^{2}\right) /\left(a_{1} \theta_{+}^{2}\right)>1$, so $x>0$ in (53). Putting (53) into $B(x)$, we have

$$
\begin{gathered}
\min _{x \in[0, \infty)} B(x)=a X_{0}^{-\gamma}\left[a_{1} \theta_{+}\left(\frac{-a_{2} \theta_{-}^{2}}{a_{1} \theta_{+}^{2}}\right)^{\theta_{+} /\left(\theta_{+}-\theta_{-}\right)}\right. \\
\left.+a_{2} \theta_{-}\left(\frac{-a_{2} \theta_{-}^{2}}{a_{1} \theta_{+}^{2}}\right)^{\theta_{-} /\left(\theta_{+}-\theta_{-}\right)}\right] \\
=a X_{0}^{-\gamma}\left(\frac{-a_{2} \theta_{-}^{2}}{a_{1} \theta_{+}^{2}}\right)^{\theta_{+} /\left(\theta_{+}-\theta_{-}\right)} \cdot \frac{a_{1} \theta_{+}\left(\theta_{-}-\theta_{+}\right)}{\theta_{-}} \\
=a\left(-\frac{\theta_{-}}{\theta_{+}}\right)^{\theta_{-} /\left(\theta_{+}-\theta_{-}\right)} \cdot\left(\frac{2 \lambda}{\mu}-\theta_{-}\right)=a M^{*} .
\end{gathered}
$$

From (52) and (54), we can complete the proof.

Remark 13. An important inequality with respect to $M^{*}$ given by (51) is $\lambda / \mu<M^{*}<2 \lambda / \mu$.

Proof. For $M^{*}$ given by (51), taking its logarithm, we can get

$$
\begin{aligned}
& \ln M^{*}=\frac{\theta_{-}}{\theta_{+}-\theta_{-}}\left(\ln \left(-\theta_{-}\right)-\ln \theta_{+}\right)+\ln \left(\frac{2 \lambda}{\mu}-\theta_{-}\right) \\
& =-\left(\frac{\mu}{2 \sqrt{\mu^{2}+2 \lambda \sigma^{2}}}+\frac{1}{2}\right)\left[\ln \left(\mu+\sqrt{\mu^{2}+2 \lambda \sigma^{2}}\right)\right. \\
& \left.-\ln \left(-\mu+\sqrt{\mu^{2}+2 \lambda \sigma^{2}}\right)\right]+\ln \left(\mu+\sqrt{\mu^{2}+2 \lambda \sigma^{2}}\right) \\
& +\frac{1}{2} \ln \left(\mu^{2}+2 \lambda \sigma^{2}\right)-\ln \mu-\ln \sigma^{2} .
\end{aligned}
$$


Let us check the derivative of $M^{*}$ on $\sigma^{2}$ as follows:

$$
\begin{aligned}
& \frac{\partial \ln M^{*}}{\partial \sigma^{2}} \\
& =-\frac{\mu}{2} \cdot\left(-\frac{1}{2}\right) \cdot\left(\mu^{2}+2 \lambda \sigma^{2}\right)^{-3 / 2} \cdot 2 \lambda \\
& \cdot \ln \left(\frac{\mu+\sqrt{\mu^{2}+2 \lambda \sigma^{2}}}{-\mu+\sqrt{\mu^{2}+2 \lambda \sigma^{2}}}\right) \\
& -\left(\frac{\mu}{2 \sqrt{\mu^{2}+2 \lambda \sigma^{2}}}+\frac{1}{2}\right) \\
& \cdot\left[\frac{\lambda\left(\mu^{2}+2 \lambda \sigma^{2}\right)^{-1 / 2}}{\mu+\sqrt{\mu^{2}+2 \lambda \sigma^{2}}}-\frac{\lambda\left(\mu^{2}+2 \lambda \sigma^{2}\right)^{-1 / 2}}{-\mu+\sqrt{\mu^{2}+2 \lambda \sigma^{2}}}\right] \\
& +\frac{\lambda\left(\mu^{2}+2 \lambda \sigma^{2}\right)^{-1 / 2}}{\mu+\sqrt{\mu^{2}+2 \lambda \sigma^{2}}}+\frac{\lambda}{\mu^{2}+2 \lambda \sigma^{2}}-\frac{1}{\sigma^{2}} \\
& =\frac{\lambda \mu}{2} \cdot\left(\mu^{2}+2 \lambda \sigma^{2}\right)^{-3 / 2} \\
& \cdot \ln \left(\frac{\mu+\sqrt{\mu^{2}+2 \lambda \sigma^{2}}}{-\mu+\sqrt{\mu^{2}+2 \lambda \sigma^{2}}}\right)>0 .
\end{aligned}
$$

Therefore, $M^{*}$ is an increasing function of $\sigma^{2}$. From $\sigma^{2} \epsilon$ $(0, \infty)$, we can take limits as $\sigma^{2} \rightarrow 0$ and $\sigma^{2} \rightarrow \infty$ to get the infimum and the supremum of $M^{*}$. For (55), it follows that

$$
\begin{aligned}
\lim _{\sigma^{2} \rightarrow 0} & \ln M^{*} \\
= & -\ln (2 \mu)+\ln (2 \mu)+\ln \mu-\ln \mu \\
& \quad+\lim _{\sigma^{2} \rightarrow 0} \ln \left(\frac{-\mu+\sqrt{\mu^{2}+2 \lambda \sigma^{2}}}{\sigma^{2}}\right)=\ln \left(\frac{\lambda}{\mu}\right), \\
\lim _{\sigma^{2} \rightarrow \infty} \ln M^{*} & -\ln \mu \\
= & \quad \lim _{\sigma^{2} \rightarrow \infty} \ln \left(\frac{\left(-\mu+\sqrt{\mu^{2}+2 \lambda \sigma^{2}}\right) \sqrt{\mu^{2}+2 \lambda \sigma^{2}}}{\sigma^{2}}\right) \\
= & \ln \left(\frac{2 \lambda}{\mu}\right) .
\end{aligned}
$$

Since the infimum of $M^{*}$ is $\lambda / \mu$ and the supremum of $M^{*}$ is $2 \lambda / \mu$, we can get $\lambda / \mu<M^{*}<2 \lambda / \mu$.
4.2. Integral Functions $I(C)$ under Different Conditions. By the convexity of $H^{C}(x)$ on $[0, \infty)$, it shows that $C H^{C}(0) \leq k$, that is, $C \leq a^{1-\gamma}(k / \gamma)^{\gamma}$, is equivalent to $\widetilde{x}^{C} \vee 0=0$. From the definition of $H^{C}(x)$, it is known that $C>a X_{0}^{-\gamma}$. We compare $a X_{0}^{-\gamma}$ to $a^{1-\gamma}(k / \gamma)^{\gamma}$ as follows.

Case $1\left(a X_{0}^{-\gamma}<a^{1-\gamma}(k / \gamma)^{\gamma}\right)$. By simplicity, this condition is equivalent to $k>2 a \lambda / \mu$. In addition, at point $C=a^{1-\gamma}(k / \gamma)^{\gamma}$, from (45) we have that $C H^{C}(0)=k$ and $\left(C H^{C}\right)^{\prime}(0)<0$. Combining with the convexity of $H^{C}(x)$, it follows that $x_{1}^{C}>$ 0 and $k-C H^{C}(x)>0$ on $\left(0, x_{1}^{C}\right)$.

Proposition 14. If $k>2 a \lambda / \mu$, then $I_{1}\left(a^{1-\gamma}(k / \gamma)^{\gamma}\right)>0$ is satisfied. Consequently, from the monotonicity of $I_{1}(C)$, we have $I_{2}\left(a X_{0}^{-\gamma} a_{1}\right)=I_{1}\left(a X_{0}^{-\gamma}\right)>0$.

From the above, it shows that $a X_{0}^{-\gamma}$ can be the left domain endpoint for $I_{1}(C)$. On the other hand, from $\mathrm{CH}^{C}(x) \rightarrow \infty$ as $C \rightarrow \infty$ and $I_{1}(C)$ is a continuous function, then there exists $C^{*}>a^{1-\gamma}(k / \gamma)^{\gamma}$, such that $I_{1}\left(C^{*}\right)=0$. Therefore, $\left(a X_{0}^{-\gamma}, C^{*}\right)$ can be the domain of $I_{1}(C)$. Moreover, we have that $\left(0, a X_{0}^{-\gamma} a_{1}\right]$ can be the domain of $I_{2}\left(C_{1}\right)$ from Section 4.1.

Remark 15. If $k>2 a \lambda / \mu$, we have $I_{2}\left(C a_{1}\right) \in\left[I_{2}\left(a X_{0}^{-\gamma} a_{1}\right), \infty\right)$, for $C \in\left(0, a X_{0}^{-\gamma}\right]$ and $I_{1}(C) \in\left(0, I_{2}\left(a X_{0}^{-\gamma} a_{1}\right)\right)$, for $C \in$ $\left(a X_{0}^{-\gamma}, C^{*}\right)$, where $C^{*}>a^{1-\gamma}(k / \gamma)^{\gamma}$.

Therefore, we can define

$$
I(C)= \begin{cases}I_{2}\left(C a_{1}\right), & C \in\left(0, a X_{0}^{-\gamma}\right], \\ I_{1}(C), & C \in\left(a X_{0}^{-\gamma}, C^{*}\right)\end{cases}
$$

as a useful integral function under $a X_{0}^{-\gamma}<a^{1-\gamma}(k / \gamma)^{\gamma}$. Moreover, $I(C)$ is a strictly decreasing function.

Case $2\left(a X_{0}^{-\gamma} \geq a^{1-\gamma}(k / \gamma)^{\gamma}\right)$. By simplicity, this assumption is equivalent to $k \leq 2 a \lambda / \mu$. In addition, in $I_{2}\left(C_{1}\right)$ the inequality $H^{C_{1}}(0) \leq k$ is equivalent to $C_{1} \leq\left(k-a \theta_{-}\right) /\left(\theta_{+}-\theta_{-}\right)$. From (22), we can obtain that $\theta_{-}^{2}\left(k-a \theta_{+}\right) / \theta_{+}^{2}\left(k-a \theta_{-}\right)>1$ is equivalent to $k>a \lambda / \mu$. Then, for $0<x<\left(1 / \theta_{+}-\right.$ $\left.\left.\theta_{-}\right)\right) \ln \left(\theta_{-}^{2}\left(k-a \theta_{+}\right) / \theta_{+}^{2}\left(k-a \theta_{-}\right)\right)$, it can be easily seen that $\left(H^{C_{1}}\right)^{\prime}(x)<0$ at $C_{1}=\left(k-a \theta_{-}\right) /\left(\theta_{+}-\theta_{-}\right)$. By the similar discussion as above, we can show that as follows.

Proposition 16. If $a \lambda / \mu<k \leq 2 a \lambda / \mu$, then $I_{2}\left(\left(k-a \theta_{-}\right) /\left(\theta_{+}-\right.\right.$ $\left.\left.\theta_{-}\right)\right)>0$. If $k \leq a \lambda / \mu$, then $I_{2}\left(\left(k-a \theta_{-}\right) /\left(\theta_{+}-\theta_{-}\right)\right)=0$ and $I_{2}\left(C_{1}\right)>0$ for any $C_{1} \in\left(0,\left(k-a \theta_{-}\right) /\left(\theta_{+}-\theta_{-}\right)\right)$.

Remark 17. If $k \leq a \lambda / \mu$, we have $I_{2}\left(C a_{1}\right) \in(0,+\infty)$ for $C \in\left(0, C^{*}\right)$, where $C^{*}=\left(k-a \theta_{-}\right) /\left(a_{1}\left(\theta_{+}-\theta_{-}\right)\right)$. If $a \lambda / \mu<$ $k \leq a M^{*}$, we have $I_{2}\left(C a_{1}\right) \in(0,+\infty)$ for $C \in\left(0, C^{*}\right)$, where $\left(k-a \theta_{-}\right) /\left(a_{1}\left(\theta_{+}-\theta_{-}\right)\right)<C^{*} \leq a X_{0}^{-\gamma}$. If $a M^{*}<k \leq 2 a \lambda / \mu$, we have $I_{2}\left(C a_{1}\right) \in\left[I_{2}\left(a X_{0}^{-\gamma} a_{1}\right), \infty\right)$, for $C \in\left(0, a X_{0}^{-\gamma}\right]$, and $I_{1}(C) \in\left(0, I_{2}\left(a X_{0}^{-\gamma} a_{1}\right)\right)$, for $C \in\left(a X_{0}^{-\gamma}, C^{*}\right)$, where $C^{*}>$ $a X_{0}^{-\gamma}$. 
Therefore, we can also use $I_{1}(C)$ and $I_{2}\left(C a_{1}\right)$ to define three types of $I(C)$ similar to (58) as useful integral functions under $a X_{0}^{-\gamma} \geq a^{1-\gamma}(k / \gamma)^{\gamma}$. Moreover, these types of $I(C)$ are strictly decreasing functions.

4.3. Compute Parameter $C$ and Numerical Examples. In the process of calculating $C$, we can follow the same steps for these two cases. By the following steps, the uncertain parameter $C$ can be obtained. Further, we can get other parameters, such as $x_{1}^{C}, x_{1}^{C_{1}}, \widetilde{x}^{C}$, and $\widetilde{x}^{C_{1}}$.

Step 1. Compare $a M^{*}$ with $k$.

Step 2. If $k>a M^{*}$, Compute $I_{2}\left(a X_{0}^{-\gamma} a_{1}\right)$, and then compare $I_{2}\left(a X_{0}^{-\gamma} a_{1}\right)$ with $K$.

(i) If $I_{2}\left(a X_{0}^{-\gamma} a_{1}\right)>K$, get $C$ on $\left(a X_{0}^{-\gamma}, \infty\right)$, such that $I_{1}(C)=K$.

(ii) If $I_{2}\left(a X_{0}^{-\gamma} a_{1}\right) \leq K$, get $C$ on $\left(0, a X_{0}^{-\gamma}\right)$, such that $I_{2}\left(C a_{1}\right)=K$.

Step 3. If $k \leq a M^{*}$, then compare $k$ with $a \lambda / \mu$ and solve $I(C)=K$.

In practical problems, $k \in(0,1)$, but the restriction for $a$ is just $a>0$. In order to satisfy $0<k<1$, for convenience, we just choose different values for $a$ and never change the values of $k, \mu, \lambda$, and $\sigma$ in the following numerical examples. The values of these parameters are selected as $\mu=0.12$, $\lambda=0.002, \sigma=1.6$, and $k=0.13$. For the value of $a>0$, we choose $a=2,4,6,8$ to satisfy four different conditions. In Figure 1, the graphs of $I(C)$ are shown, and both forms of $I(C), I_{1}(C)$, and $I_{2}\left(C a_{1}\right)$ are listed out in their corresponding domains. Moreover, the right domain endpoint $C^{*}$ of $I(C)$ is also presented. Figure 1 shows that $I(C)$ is always a decreasing function.

In Figure 1(a), $a=2$, which satisfies $k>2 a \lambda / \mu$. We let $C_{1}^{*} / a_{1}=a X_{0}^{-\gamma}$ and $C_{0}^{*}=a^{1-\gamma}(k / \gamma)^{\gamma}$. We can see $I(C)$ is composed of $I_{2}\left(C a_{1}\right)$ for $C \in\left(0, C_{1}^{*} / a_{1}\right]$ and $I_{1}(C)$ for $C \in$ $\left(C_{1}^{*} / a_{1}, C^{*}\right)$ with $I_{2}\left(C_{1}^{*}\right)=5.2494>0$ and $I_{1}\left(C_{0}^{*}\right)=3.5408>$ 0 . This phenomenon is consistent with Proposition 14. In Figure $1(\mathrm{~b}), a=4$, which satisfies $a M^{*}<k \leq 2 a \lambda / \mu$, and $C_{0}^{*}$ is given by $C_{0}^{*}=a X_{0}^{-\gamma}$. Since $\left(k-a \theta_{-}\right) /\left(a_{1}\left(\theta_{+}-\theta_{-}\right)\right)=1.3935$ is very close to $C_{0}^{*}=1.4018$, we just draw the position of $C_{0}^{*}$ in the graph. In fact, under $a M^{*}<k \leq 2 a \lambda / \mu$, this phenomenon happens almost on any other trial, for which we select different $k, \mu, \lambda$, and $\sigma$. Figure $1(\mathrm{~b})$ shows $I_{2}\left(C_{0}^{*} a_{1}\right)>0$, which supports Proposition 12 correctly. In Figure 1(c), we choose $a=6$, which satisfies $a \lambda / \mu<k \leq a M^{*} . I(C)$ is just composed of $I_{2}\left(C a_{1}\right)$ with $I_{2}\left(\left(k-a \theta_{-}\right) /\left(\theta_{+}-\theta_{-}\right)\right)=0.1631>$ 0 , which matches Proposition 16 very well. Since the value 0.1631 , compared with the numbers of $y$-axis, is so small, it is not obvious in the graph. In Figure $1(\mathrm{~d}), a=8$, which satisfies $k \leq a \lambda / \mu$. We can obtain $\left(k-a \theta_{-}\right) /\left(a_{1}\left(\theta_{+}-\theta_{-}\right)\right)=2.4651$ by calculation. From numerical simulation we get $C^{*}=2.4651$, so $C^{*}=\left(k-a \theta_{-}\right) /\left(a_{1}\left(\theta_{+}-\theta_{-}\right)\right)$is satisfied. Therefore, the conclusion in Proposition 16 is shown.
Table 1 summarizes the results for different parameter values. As shown in Table 1 (starting from row 4), for each case in Figure 1 where $a>0$, we choose three different values of $K$ to calculate parameters such as $C, \tilde{x} \vee 0, x_{0} \vee 0$, and $x_{1}$ from $I(C)=K$. For Figure 1 (a) with $a=2$, from $I_{2}\left(C_{1}^{*}\right)=$ 5.2494 and $I_{1}\left(C_{0}^{*}\right)=3.5408$, three potential values of $K$ are selected as $K=0.15<I_{1}\left(C_{0}^{*}\right), I_{1}\left(C_{0}^{*}\right)<K=4<I_{2}\left(C_{1}^{*}\right)$, and $K=8>I_{2}\left(C_{1}^{*}\right)$. By the same selection method, for Figure $1(\mathrm{~b})$ with $a=4$, from $I_{2}\left(\left(k-a \theta_{-}\right) /\left(\theta_{+}-\theta_{-}\right)\right)=1.0669$ and $I_{2}\left(C_{0}^{*} a_{1}\right)=1.0150$, three trials for $K$ are $K=0.15,1.05,4$. For Figure $1(\mathrm{c})$ with $a=6$, from $I_{2}\left(\left(k-a \theta_{-}\right) /\left(\theta_{+}-\theta_{-}\right)\right)=$ 0.1631 , three comparable values of $K$ are given by $K=$ $0.15,1.05,4$ and the same is applied in Figure $1(\mathrm{~d})$ with $a=8$. This table shows that the results, whether the values of $\tilde{x} \vee 0$ and $x_{0} \vee 0$ are zeroes or not, are totally consistent with the theoretical results in Sections 4.1 and 4.2. In addition, $C, \tilde{x} \vee 0$, and $x_{0} \vee 0$ are decreasing and $x_{1}$ is increasing when $K$ is increasing under the same $a$. On the other hand, under the same $K, C$ is increasing and $x_{1}$ is decreasing as $a$ is increasing.

\section{Solutions to the QVI and the Optimal Policy}

Notice that all possible structures to calculate $C$ are $I_{1}(C)$ and $I_{2}\left(\mathrm{Ca}_{1}\right)$. Moreover, all integrands of these structures can be used to construct the candidate solutions to the QVI. In the following, we first demonstrate that all constructed candidate solutions are solutions to the QVI under different conditions and give several corresponding numerical examples by using the results of Section 4.3. Then, we provide and verify the optimal policy based on the solutions to the QVI.

5.1. Solutions to the QVI and Numerical Examples. Assume that we have got $C$, such that $I(C)=K$, by those steps provided in Section 4.3. Then, other parameters, such as $x_{1}^{C}$, $x_{1}^{C_{1}}, \widetilde{x}^{C} \vee 0$, and $\widetilde{x}^{C_{1}} \vee 0$, can also be obtained. For simplicity, we use the same denotations as before, such as $C, C_{1}, x_{1}^{C}, x_{1}^{C_{1}}$, $\widetilde{x}^{C} \vee 0$, and $\widetilde{x}^{C_{1}} \vee 0$, to represent all calculated parameters.

For $C$, such that $I_{1}(C)=K$, we define a function $T_{1}(x)$ by

$$
T_{1}(x)= \begin{cases}C H^{C}(x), & x \in\left[0, x_{1}^{C}\right), \\ k, & x \in\left[x_{1}^{C}, \infty\right) .\end{cases}
$$

Then $C>a X_{0}^{-\gamma}$ and $T_{1}(x)$ satisfy $I_{1}(C)=K$.

Define $v_{1}(x)=a+\int_{0}^{x} T_{1}(y) d y$; then we can obtain that

$v_{1}(x)$

$$
= \begin{cases}C\left[x+\left(\frac{a}{C}\right)^{1 / \gamma}\right]^{\gamma}, & x \in\left[0, x_{0}\right), \\ C a_{1} e^{\theta_{+}\left(x-x_{0}\right)}+C a_{2} e^{\theta_{-}\left(x-x_{0}\right)}, & x \in\left[x_{0}, x_{1}^{C}\right), \\ v\left(x_{1}^{C}\right)+k\left(x-x_{1}^{C}\right), & x \in\left[x_{1}^{C}, \infty\right),\end{cases}
$$

where $x_{0}=X_{0}-(a / C)^{1 / \gamma}$.

In addition, for $C$, such that $I_{2}\left(C a_{1}\right)=K$, define

$$
T_{2}(x)= \begin{cases}H^{C_{1}}(x), & x \in\left[0, x_{1}^{C_{1}}\right), \\ k, & x \in\left[x_{1}^{C_{1}}, \infty\right),\end{cases}
$$




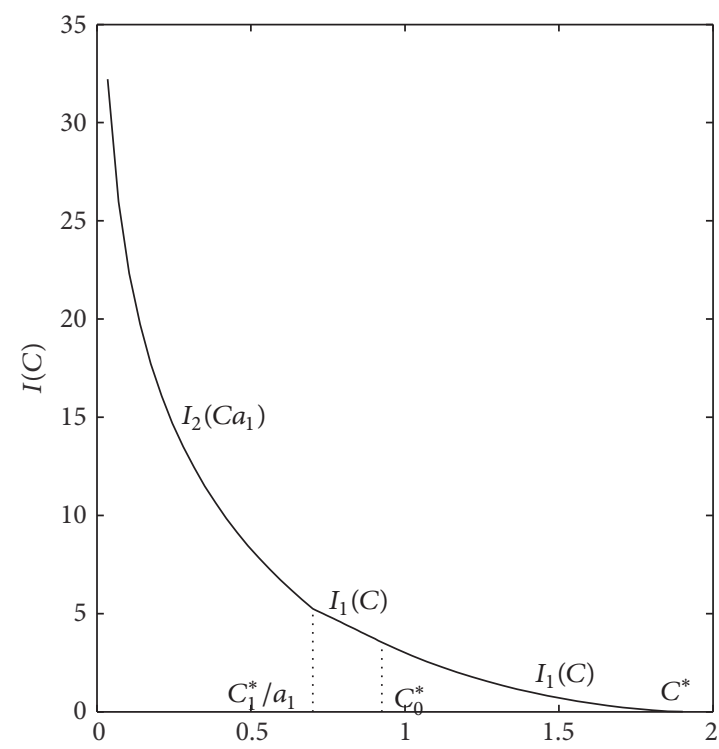

(a) $a=2$

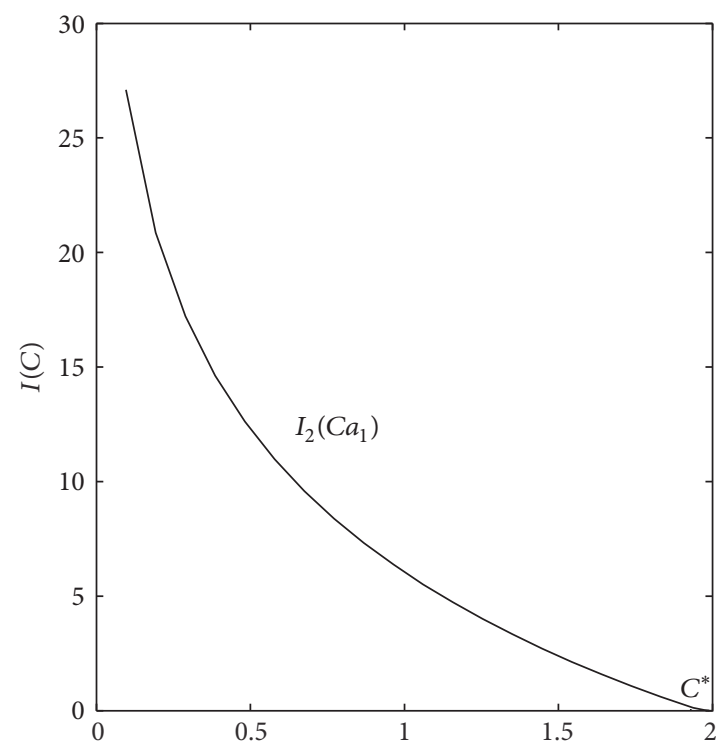

(c) $a=6$

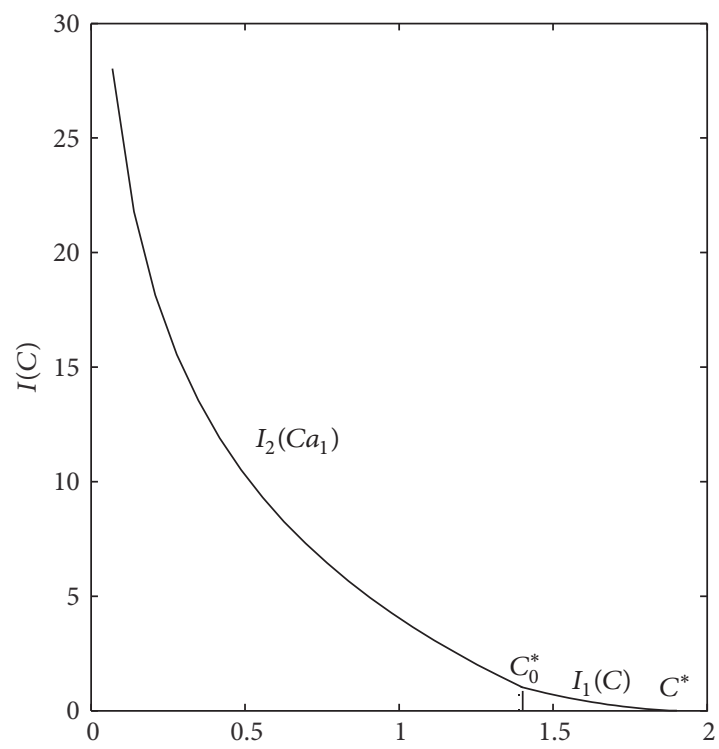

(b) $a=4$

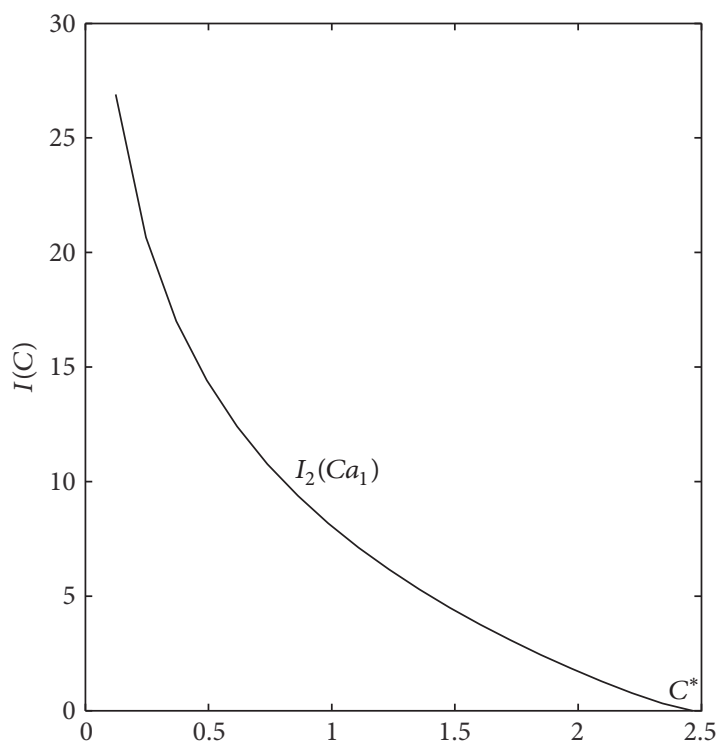

(d) $a=8$

FIgURE 1: $I(C)$ with respect to $a$.

where $C_{1}=C a_{1}$. Then $C \leq a X_{0}^{-\gamma}$ and $T_{2}(x)$ satisfy $I_{2}\left(C_{1}\right)=$ $K$.

Define $v_{2}(x)=a+\int_{0}^{x} T_{2}(y) d y$; it follows that

$$
v_{2}(x)= \begin{cases}C_{1} e^{\theta_{+} x}+\left(a-C_{1}\right) e^{\theta_{-} x}, & x \in\left[0, x_{1}^{C_{1}}\right), \\ v\left(x_{1}^{C_{1}}\right)+k\left(x-x_{1}^{C_{1}}\right), & x \in\left[x_{1}^{C_{1}}, \infty\right) .\end{cases}
$$

In the following, for $v_{1}(x)$ and $v_{2}(x)$, we use the same notations, $x_{1}$, to denote both $x_{1}^{C}$ and $x_{1}^{C_{1}}$ and $\tilde{x}$ to denote both $\widetilde{x}^{C}$ and $\widetilde{x}^{C_{1}}$. From the construction of $v_{1}(x)$ and $v_{2}(x)$, we have the following theorem by verifying the system QVI.

Theorem 18. Both functions $v_{1}(x)$ and $v_{2}(x)$ given by (60) and (62) are continuously differentiable on $[0, \infty)$, and for each of $v_{1}$ and $v_{2}$, there exists $U>0$ such that this function is twice continuously differentiable on $[0, U)$. Moreover, $v_{1}(x)$ and $v_{2}(x)$ provide a solution to $Q V I$.

In addition, from the discussion of Section 4, we have an important result as follows to make a decision with respect to whether there is a bankruptcy or not.

(1) Under the assumption $k>2 a \lambda / \mu$, if $I_{1}\left(a^{1-\gamma}(k / \gamma)^{\gamma}\right) \leq$ $K$, then $\tilde{x} \vee 0=0$ and there would be a bankruptcy. If $I_{1}\left(a^{1-\gamma}(k / \gamma)^{\gamma}\right)>K$, then $\tilde{x}>0$ and the optimal policy is continuation strategy.

(2) Under the assumption $a \lambda / \mu<k \leq 2 a \lambda / \mu$, if $I_{2}((k-$ $\left.\left.a \theta_{-}\right) /\left(\theta_{+}-\theta_{-}\right)\right) \leq K$, then $\tilde{x} \vee 0=0$ and there would 
TABLE 1: Parameter estimations under $\mu=0.12, \lambda=0.002, k=0.13$, and $\sigma=1.6$.

\begin{tabular}{|c|c|c|c|c|c|c|c|}
\hline Conditions & $a$ & $K$ & $C$ & $\tilde{x} \vee 0$ & $x_{0} \vee 0$ & $x_{1}$ & $I(C)$ \\
\hline$k>\frac{2 a \lambda}{\mu}$ & 0 & 0.15 & 1.5748 & 15.9820 & 12.4675 & 49.9571 & $I_{0}(C)$ \\
\hline$k>\frac{2 a \lambda}{\mu}$ & 0 & 4 & 0.8671 & 5.7239 & 12.4675 & 91.9615 & $I_{0}(C)$ \\
\hline$k>\frac{2 a \lambda}{\mu}$ & 0 & 8 & 0.5196 & 2.3827 & 12.4675 & 127.4305 & $I_{0}(C)$ \\
\hline$k>\frac{2 a \lambda}{\mu}$ & 2 & 0.15 & 1.7489 & 18.4531 & 11.0865 & 40.0506 & $I_{1}(C)$ \\
\hline$k>\frac{2 a \lambda}{\mu}$ & 2 & 4 & 0.8648 & 0 & 4.9491 & 84.6289 & $I_{1}(C)$ \\
\hline$k>\frac{2 a \lambda}{\mu}$ & 2 & 8 & 0.5163 & 0 & 0 & 115.3948 & $I_{2}\left(C a_{1}\right)$ \\
\hline$a M^{*}<k \leq \frac{2 a \lambda}{\mu}$ & 4 & 0.15 & 1.7490 & 12.5186 & 5.1482 & 34.1052 & $I_{1}(C)$ \\
\hline$a M^{*}<k \leq \frac{2 a \lambda}{\mu}$ & 4 & 1.05 & 1.3962 & 0.1815 & 0 & 46.2875 & $I_{2}\left(C a_{1}\right)$ \\
\hline$a M^{*}<k \leq \frac{2 a \lambda}{\mu}$ & 4 & 4 & 1.0055 & 0 & 0 & 69.2676 & $I_{2}\left(C a_{1}\right)$ \\
\hline$\frac{a \lambda}{\mu}<k \leq a M^{*}$ & 6 & 0.15 & 1.9325 & 0.4645 & 0 & 22.0481 & $I_{2}\left(C a_{1}\right)$ \\
\hline$\frac{a \lambda}{\mu}<k \leq a M^{*}$ & 6 & 1.05 & 1.7409 & 0 & 0 & 31.7341 & $I_{2}\left(C a_{1}\right)$ \\
\hline$\frac{a \lambda}{\mu}<k \leq a M^{*}$ & 6 & 4 & 1.2550 & 0 & 0 & 54.2053 & $I_{2}\left(C a_{1}\right)$ \\
\hline$k \leq \frac{a \lambda}{\mu}$ & 8 & 0.15 & 2.3953 & 0 & 0 & 11.0653 & $I_{2}\left(C a_{1}\right)$ \\
\hline$k \leq \frac{a \lambda}{\mu}$ & 8 & 1.05 & 2.1493 & 0 & 0 & 21.6008 & $I_{2}\left(C a_{1}\right)$ \\
\hline$k \leq \frac{a \lambda}{\mu}$ & 8 & 4 & 1.5614 & 0 & 0 & 40.7502 & $I_{2}\left(C a_{1}\right)$ \\
\hline
\end{tabular}

be a bankruptcy. If $I_{2}\left(\left(k-a \theta_{-}\right) /\left(\theta_{+}-\theta_{-}\right)\right)>K$, then $\tilde{x}>0$ and the optimal policy is continuation strategy.

(3) If $k \leq a \lambda / \mu$, then $\tilde{x} \vee 0=0$ and there would be a bankruptcy.

Based on the calculated parameters, $C, x_{0} \vee 0$, and $x_{1}$ of Section 4.3, several numerical examples are provided here to support our theoretical results. In Figure 2, the graphs of $v(x)$ are shown under $a=2, a=4, a=6$, and $a=8$, respectively. These figures show that at the same value of $a$, the graphs of $v(x)$ with greater $K$ are below those of $v(x)$ with smaller $K$. This phenomenon means that the optimal dividends would be greater if the fixed costs become smaller, which accords with the actual situation in the real world. Moreover, given $x$, the value of the firm $v(x)$ increases with the recovery rate $a$.

In Figure 3, the corresponding derivatives of $v(x)$ in Figure 2 are plotted. For Figure $3(\mathrm{a})$ at $K=0.15$, Figure $3(\mathrm{~b})$ at $K=0.15$ and $K=1.05$, and Figure 3(c) at $K=0.15$, it shows that they have $v^{\prime}(0)>k$ in corresponding graphs. In addition, for Figure 3 (b) at $K=1.05$, the graph of it has convexity. Moreover, for Figure 3(b) at $K=4$, Figure 3(c) at
$K=1.05$ and $K=4$, and Figure $3(\mathrm{~d})$ at $K=0.15, K=1.05$, and $K=4$, all graphs of them are below the level line of $k$.

For comparative purposes, we also compute the results for the cases when $a=0$, which are considered by Cadenillas et al. [14]. The first three rows in Table 1 present the cases for $a=0$. As shown, the results for $a=0$ are quite different from those for $a \neq 0$ (see rows $4-15$ of Table 1). An important difference is that, for $a=0, x_{0}$ and $\tilde{x}$ are all greater than zero, which are critical factors to reduce the complexity of verification for the uniqueness of solutions. The value of $v(x)$ and its derivatives under $a=0$ are plotted in Figure 4 . In fact, in Figure 4(a) $v(x)$ is the lower limit lines of those corresponding lines with the same parameters except for $a$ in Figure 2 as $a \rightarrow 0$. Comparing Figure 4(b) with Figure 3, it shows that the case $a=0$ has much simpler graphs than the case $a \neq 0$ for the derivative function $v^{\prime}(x)$. The former only has a convex shape, whereas the latter has both convex and concave shapes, which are consistent with the complicated properties of $I(C)$ in Section 4.2. More importantly, for any given $x$, the value of the firm $v(x)$ is always lower in the case with no recovery $(a=0)$ than that with a recovery 

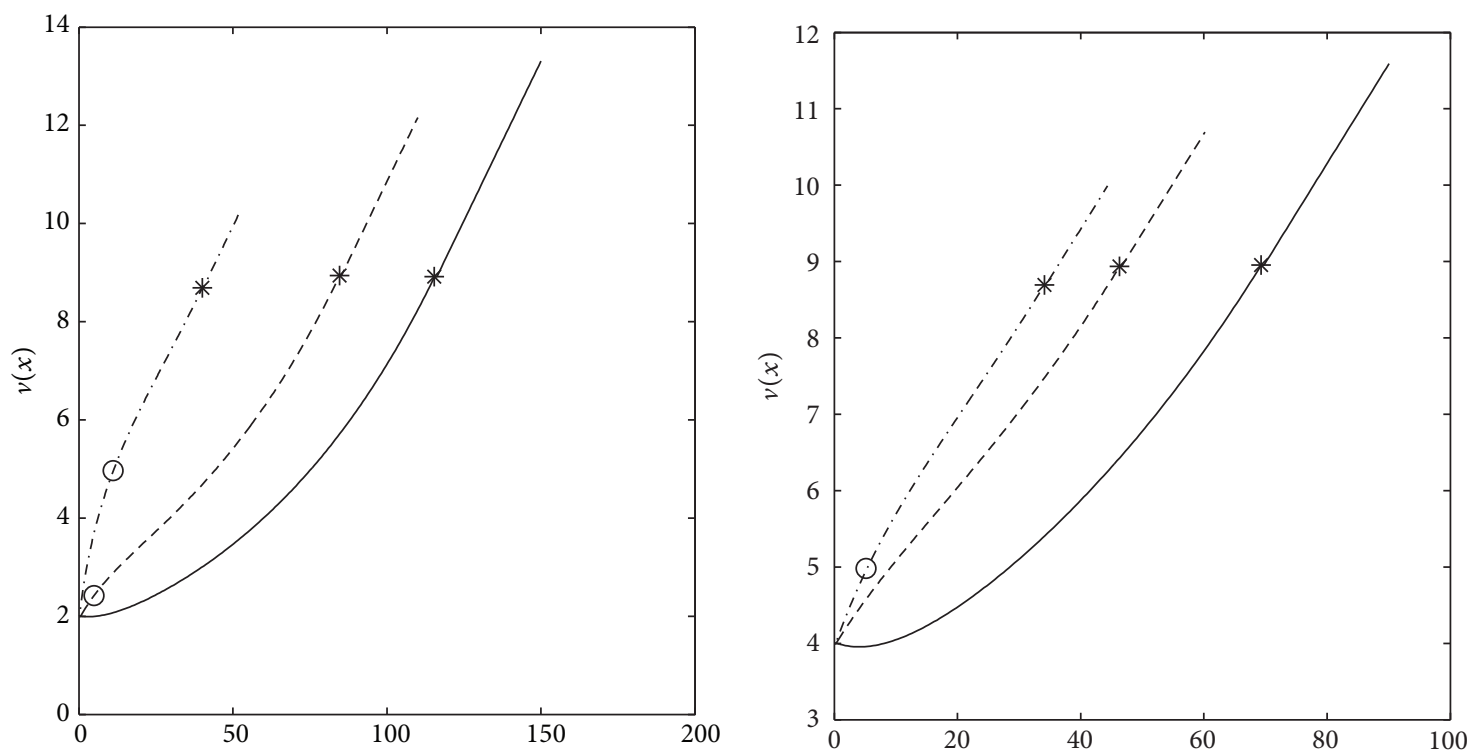

$$
\begin{aligned}
-\cdot-K & =0.15 \\
---K & =4.00 \\
-K & =8
\end{aligned}
$$

$$
\begin{aligned}
--K & =0.15 \\
--K & =1.05 \\
-K & =4
\end{aligned}
$$

(b) $a=4$

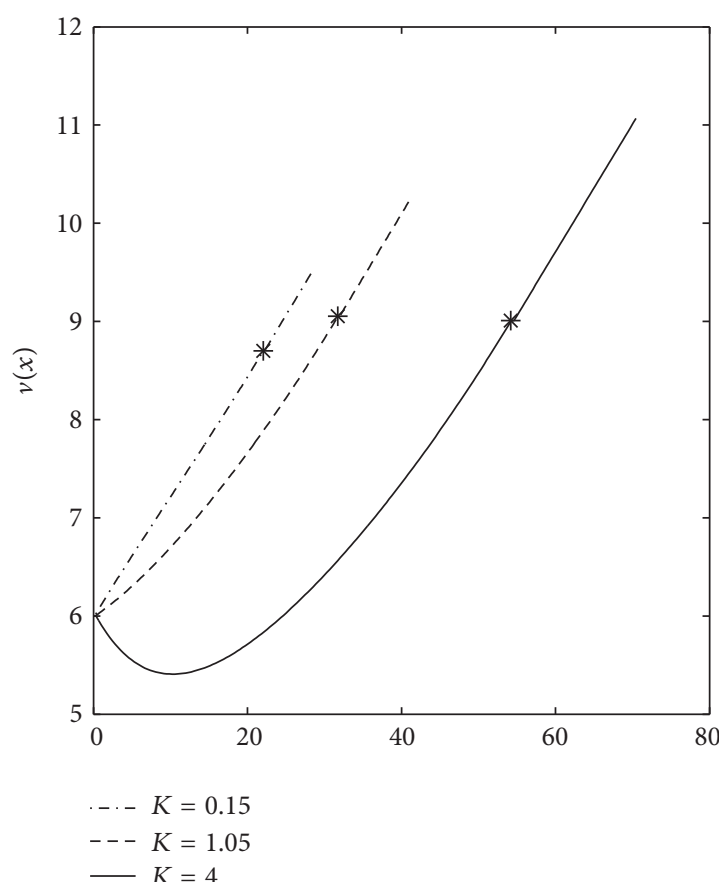

(c) $a=6$

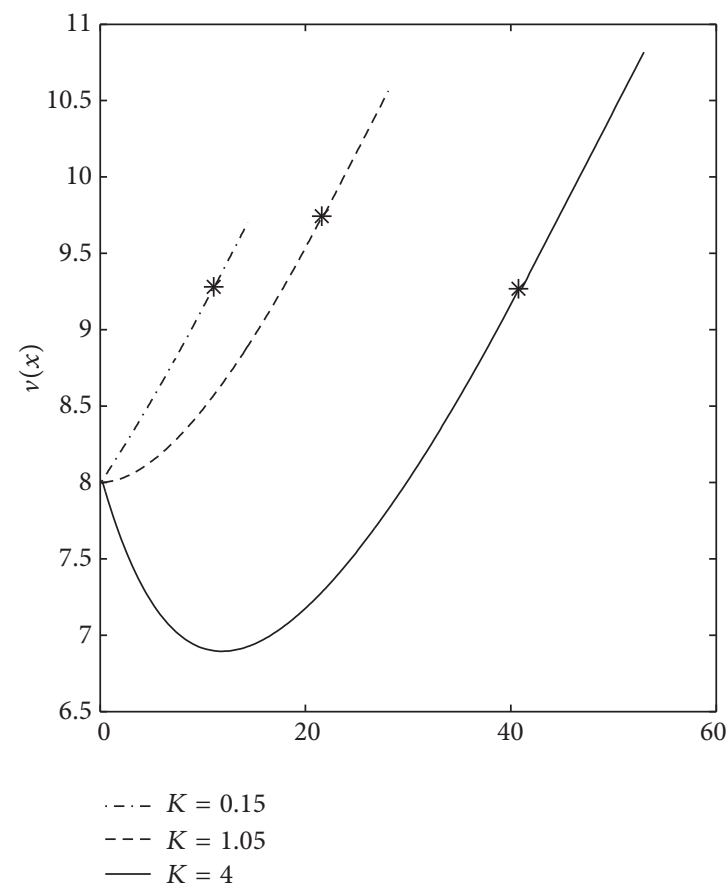

(d) $a=8$

FIgURE 2: $v(x)$ with respect to $a$.

value $(a \neq 0)$. Results show that the model with $a=0$ underestimates the firm value when there is a residual value at bankruptcy.

5.2. Optimal Policy. In the following theorem, the optimal policy is presented and verified, and it is shown that the solution to QVI constructed above is the value function.
Theorem 19. Let $\tau_{0}=0$; then the control $\pi^{*}=\left(u^{*}, \mathscr{T}^{*}, \xi^{*}\right.$, $\left.\tau^{*}\right)$ defined by

$$
\begin{aligned}
& u^{*}(t) \\
& = \begin{cases}\frac{\mu}{(1-\gamma) \sigma^{2}}\left[X^{*}+\left(\frac{a}{C}\right)^{1 / \gamma}\right], & X^{*}(t) \in\left[0, x_{0} \vee 0\right), \\
1, & X^{*}(t) \in\left[x_{0} \vee 0, \infty\right),\end{cases}
\end{aligned}
$$



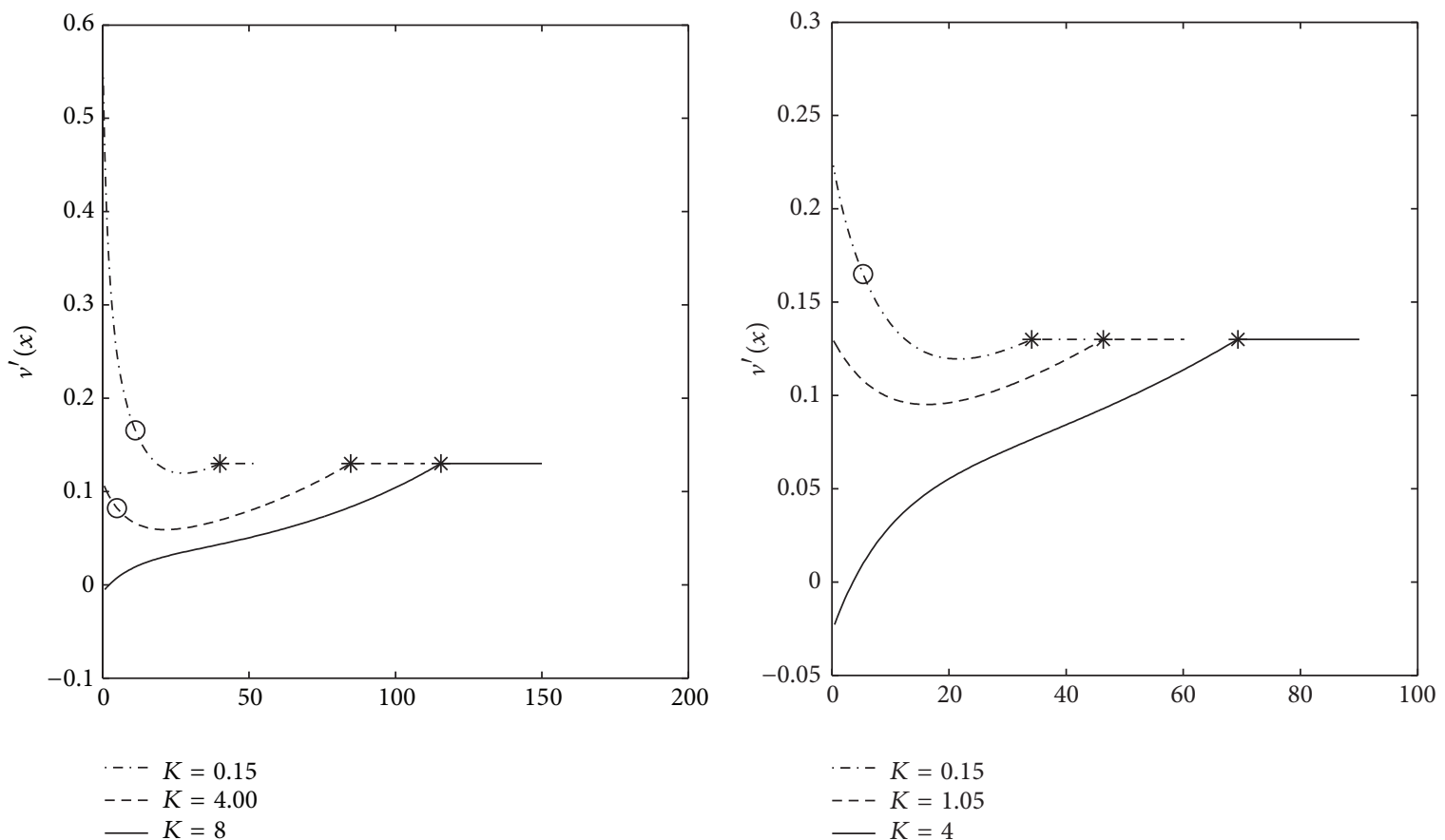

(a) $a=2, k=0.13$

(b) $a=4, k=0.13$

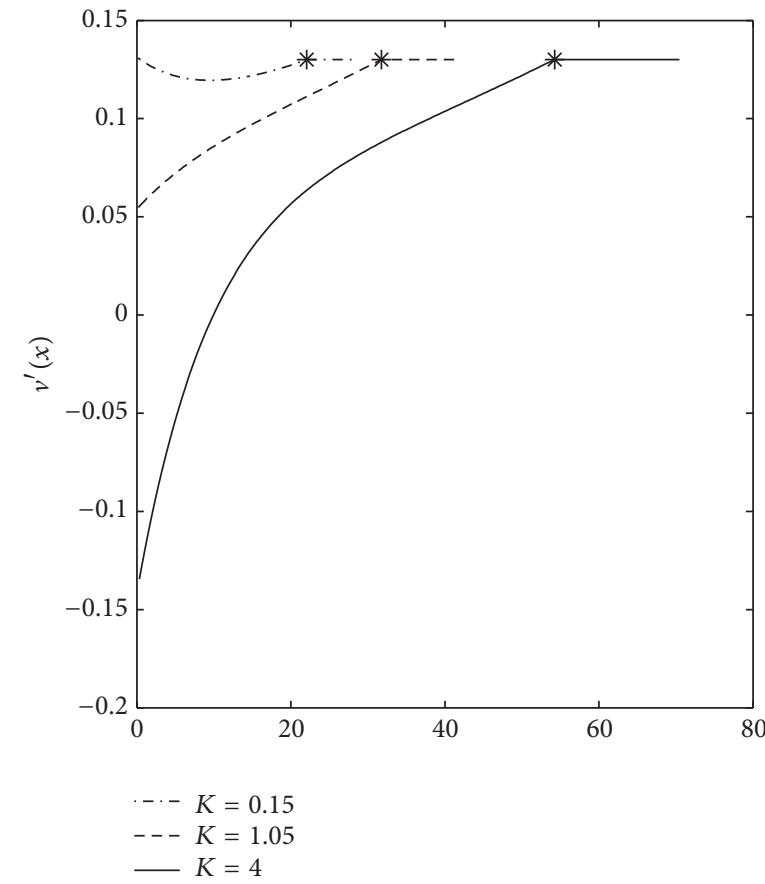

(c) $a=6, k=0.13$

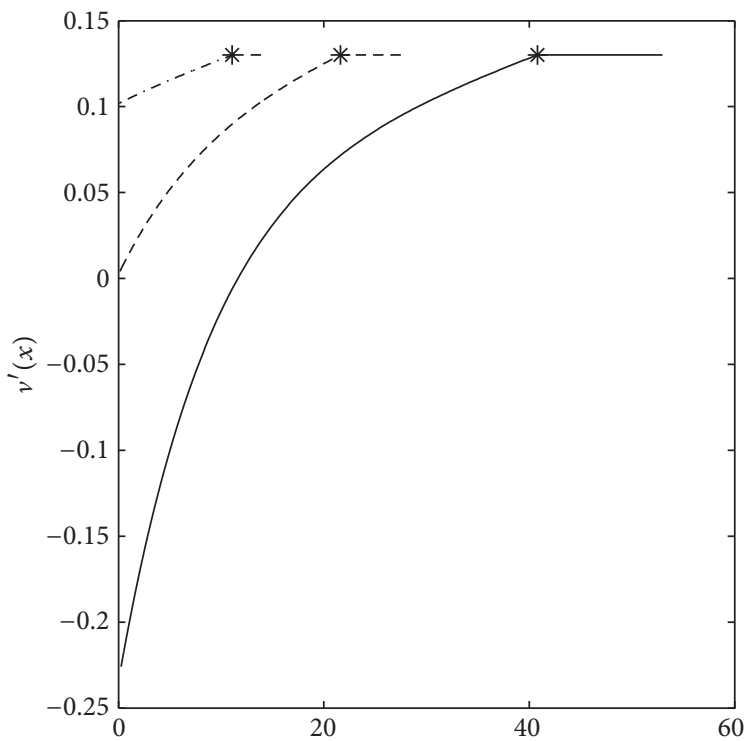

$$
\begin{aligned}
-\cdot-K & =0.15 \\
---K & =1.05 \\
-K & =4
\end{aligned}
$$

(d) $a=8, k=0.13$

FIGURE 3: $v^{\prime}(x)$ with respect to $a$.

$\tau^{*}=\inf \left\{t \geq 0: X^{*}(t)=0\right\}$,

$\tau_{1}^{*}=\inf \left\{t \geq 0: X^{*}(t)=x_{1}\right\}$

$\xi_{1}^{*}=x_{1}-\tilde{x} \vee 0$

if $\tilde{x} \vee 0=0$, then let $\tau_{n}^{*}=\infty$ and $\xi_{n}^{*}=0$; if $\tilde{x} \vee 0>0$, then every $n \geq 2$,

$$
\begin{aligned}
& \tau_{n}^{*}=\inf \left\{t \geq \tau_{n-1}: X^{*}(t)=x_{1}\right\}, \\
& \xi_{n}^{*}=x_{1}-\tilde{x},
\end{aligned}
$$

where $X^{*}$ is the solution to the stochastic differential equation.

$$
X^{*}(t)=X^{*}(0)+\int_{0}^{t} \mu u^{*}\left(X^{*}(s)\right) d s
$$




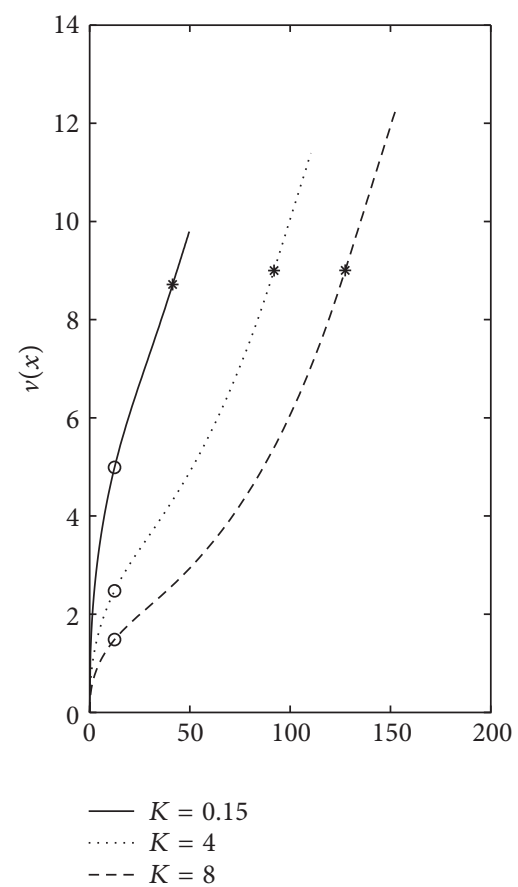

(a) $a=0$

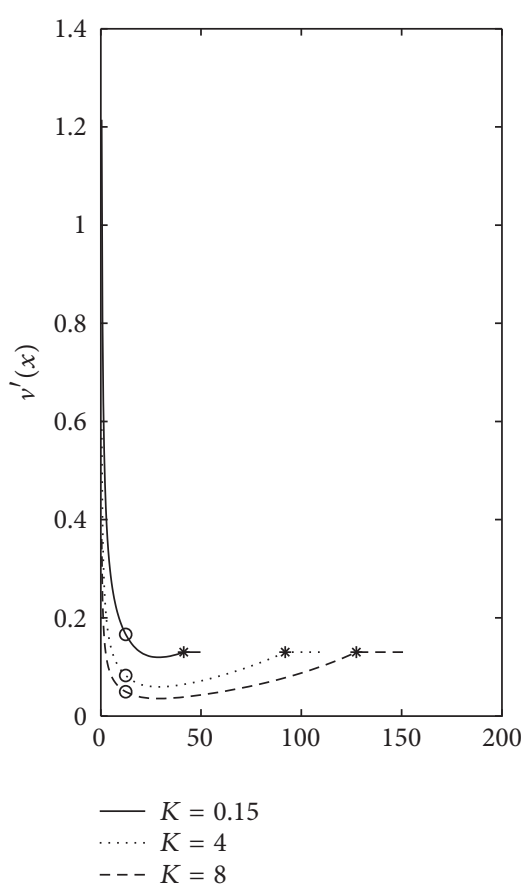

(b) $a=0$

Figure 4: $v(x)$ and $v^{\prime}(x)$ under $a=0$.

$$
\begin{aligned}
& +\int_{0}^{t} \sigma u^{*}\left(X^{*}(s)\right) d W_{s} \\
& -\left(x_{1}-\tilde{x} \vee 0\right) \sum_{n=1}^{\infty} I_{\left\{\tau_{n}^{*}<t\right\}}
\end{aligned}
$$

is the QVI control associated with the functions $v_{1}$ defined by (60) and $v_{2}$ defined by (62). This control is optimal and the functions $v_{1}(x)$ and $v_{2}(x)$ coincide with the value function.

Proof. In Section 5.1, for any functions $v_{1}$ defined by (60) and $v_{2}$ defined by (62), we can see that $v_{1}(x)$ and $v_{2}(x)$ satisfy all conditions of Theorem 5 . From Definition 3 and the discussion in Sections 3 and 4, we show that the control $\pi^{*}$ defined in (63)-(65) is the control associated with $v_{1}$ and $v_{2}$. In addition, the control $\pi^{*}$ is admissible from Definition 1 . So, by Theorem 18 , the claim that $v_{1}(x)$ and $v_{2}(x)$ are the value functions and $\pi^{*}$ is the optimal policy is proved.

\section{Conclusion}

In this paper, we consider the dividend optimization problem that some fixed money would be returned to shareholders at the state of terminal bankruptcy. For this problem, the optimal timing and the optimal amount of dividends paid out to the shareholders and the resulting firm values are provided. Moreover, we identify some conditions that there would be a terminal bankruptcy under the optimal policy. A challenge in this paper is that the structure of the candidate solution is not explicit since the existing interval of it has unfixed endpoints, which depends on some unknown parameters. We overcome this difficulty by using the integral, $I(C)$, and dividing it into several cases. The importance of this paper is that it generalizes the terminal bankruptcy model and provides several new kinds of optimal dividend strategies, especially at the state of bankruptcy.

In the future, we can address some problems with general drift and diffusion terms under the assumption that shareholders can get some fixed money when there is terminal bankruptcy. For this kind of problem, sometimes we cannot write out the explicit expression for the solutions. Then we need to use some suitable numerical methods to simulate some nonlinear equations in the quasi-variational inequalities. Moreover, we can compare two optimal policies derived from two choices, investing new money or terminating the business, and, based on the obtained result, the best corporate decision can be determined.

\section{Disclosure}

The authors declare that this paper is revised and based on Dr. Peimin Chen's dissertation presented to University of Missouri.

\section{Competing Interests}

The authors declare that there is no conflict of interests regarding the publication of this paper. 


\section{Acknowledgments}

The work on this paper was supported by the Funds for "1000 Talents Plan" of Sichuan Province (Fund number listed in Southwestern University of Finance and Economics is 221410001003040002).

\section{References}

[1] M. H. Miller and F. Modigliani, "Dividend policy, growth, and the valuation of shares," The Journal of Business, vol. 34, no. 4, pp. 411-433, 1961.

[2] M. H. Miller and K. Rock, "Dividend policy under asymmetric information," The Journal of Finance, vol. 40, no. 4, pp. 1031-1051, 1985.

[3] M. C. Jensen, "Agency cost of free cash flow, corporate finance, and takeovers," The American Economic Review, vol. 76, no. 2, pp. 323-329, 1986.

[4] M. Belhaj, "Optimal dividend payments when cash reserves follow a jump-diffusion process," Mathematical Finance, vol. 20, no. 2, pp. 313-325, 2010.

[5] C.-F. Lee, M. C. Gupta, H.-Y. Chen, and A. C. Lee, "Optimal payout ratio under uncertainty and the flexibility hypothesis: theory and empirical evidence," Journal of Corporate Finance, vol. 17, no. 3, pp. 483-501, 2011.

[6] R. Radner and L. Shepp, "Risk vs. profit potential: a model for corporate strategy," Journal of Economic Dynamics and Control, vol. 20, no. 8, pp. 1373-1393, 1996.

[7] S. Asmussen and M. Taksar, "Controlled diffusion models for optimal dividend pay-out," Insurance: Mathematics and Economics, vol. 20, no. 1, pp. 1-15, 1997.

[8] J. Paulsen and H. k. Gjessing, "Optimal choice of dividend barriers for a risk process with stochastic return on investments," Insurance: Mathematics \& Economics, vol. 20, no. 3, pp. 215-223, 1997.

[9] P. Boyle, R. J. Elliott, and H. Yang, Controlled Diffusion Models of an Insurance Company, Department of Statistics, The University of Hong Kong, 1998.

[10] B. Højgaard and M. Taksar, "Optimal proportional reinsurance policies for diffusion models with transaction costs," Insurance: Mathematics and Economics, vol. 22, no. 1, pp. 41-51, 1998.

[11] B. Højgaard and M. Taksar, "Optimal proportional reinsurance policies for diffusion models," Scandinavian Actuarial Journal, vol. 1998, no. 2, pp. 166-180, 1998.

[12] M. I. Taksar and X. Y. Zhou, "Optimal risk and dividend control for a company with a debt liability," Insurance: Mathematics and Economics, vol. 22, no. 1, pp. 105-122, 1998.

[13] F. Hubalek and W. Schachermayer, "Optimizing expected utility of dividend payments for a Brownian risk process and a peculiar nonlinear ODE," Insurance: Mathematics \& Economics, vol. 34, no. 2, pp. 193-225, 2004.

[14] A. Cadenillas, T. Choulli, M. Taksar, and L. Zhang, "Classical and impulse stochastic control for the optimization of the dividend and risk policies of an insurance firm," Mathematical Finance, vol. 16, no. 1, pp. 181-202, 2006.

[15] J. Paulsen, "Optimal dividend payments until ruin of diffusion processes when payments are subject to both fixed and proportional costs," Advances in Applied Probability, vol. 39, no. 3, pp. 669-689, 2007.

[16] J. Paulsen, "Optimal dividend payments and reinvestments of diffusion processes with both fixed and proportional costs,"
SIAM Journal on Control and Optimization, vol. 47, no. 5, pp. 2201-2226, 2008.

[17] B. Avanzi and B. Wong, "On a mean reverting dividend strategy with Brownian motion," Insurance: Mathematics \& Economics, vol. 51, no. 2, pp. 229-238, 2012.

[18] M. Jeanblanc-Picqué and A. N. Shiryaev, "Optimization of the flow of dividends," Russian Mathematical Surveys, vol. 50, no. 2, pp. 257-277, 1995.

[19] D. Liu and Z. Liu, "Dividend problems with a barrier strategy in the dual risk model until bankruptcy," Journal of Applied Mathematics, vol. 2014, Article ID 184098, 7 pages, 2014.

[20] J. Eisenberg, "Optimal dividends under a stochastic interest rate," Insurance: Mathematics and Economics, vol. 65, no. 4, pp. 259-266, 2015.

[21] D. Yao, R. Wang, and L. Xu, "Optimal impulse control for dividend and capital injection with proportional reinsurance and exponential premium principle," Communications in Statistics. Theory and Methods, vol. 46, no. 5, pp. 2519-2541, 2017.

[22] S. Sethi and M. Taksar, "Infinite-horizon investment consumption model with a nonterminal bankruptcy," Journal of Optimization Theory and Applications, vol. 74, no. 2, pp. 333346, 1992. 


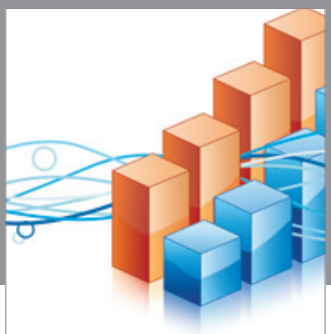

Advances in

Operations Research

vatem alat4

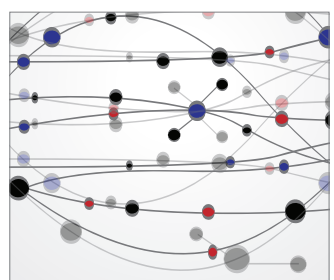

\section{The Scientific} World Journal
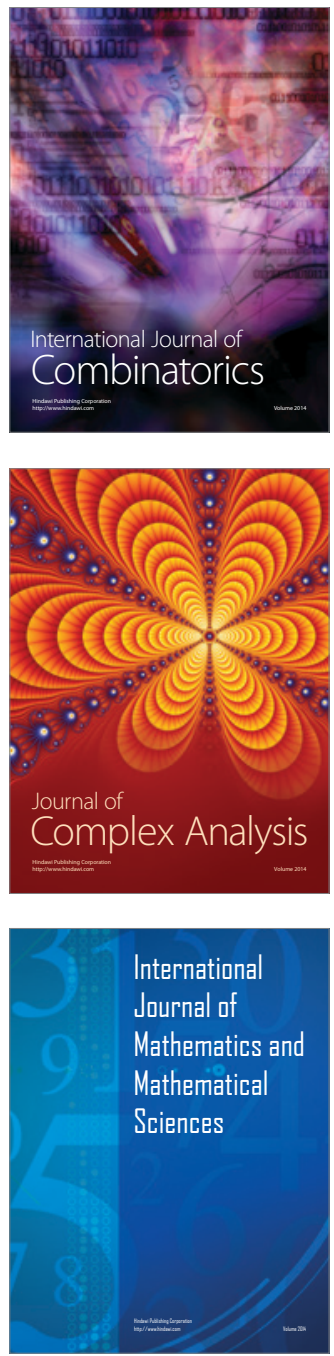
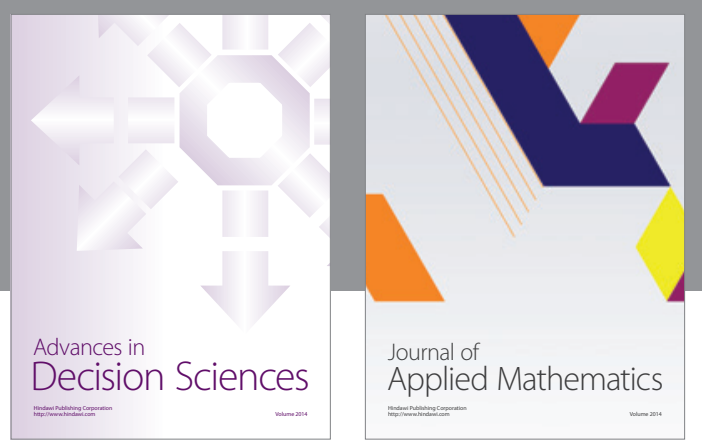

Algebra

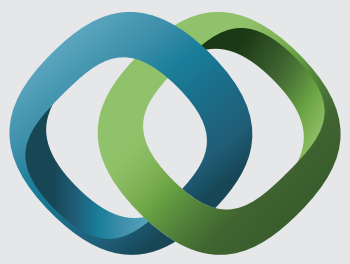

\section{Hindawi}

Submit your manuscripts at

https://www.hindawi.com
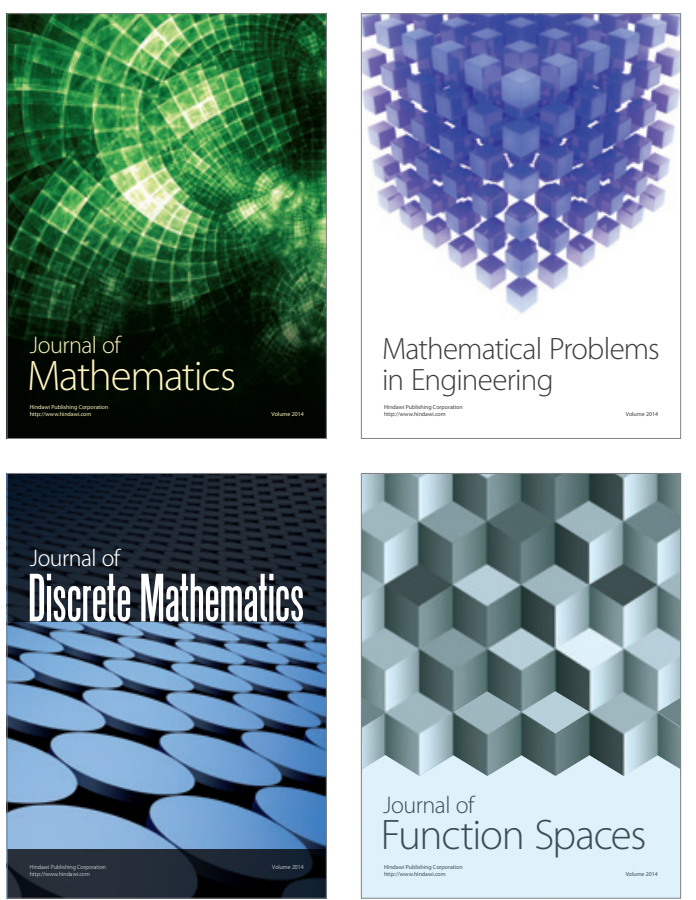

Mathematical Problems in Engineering
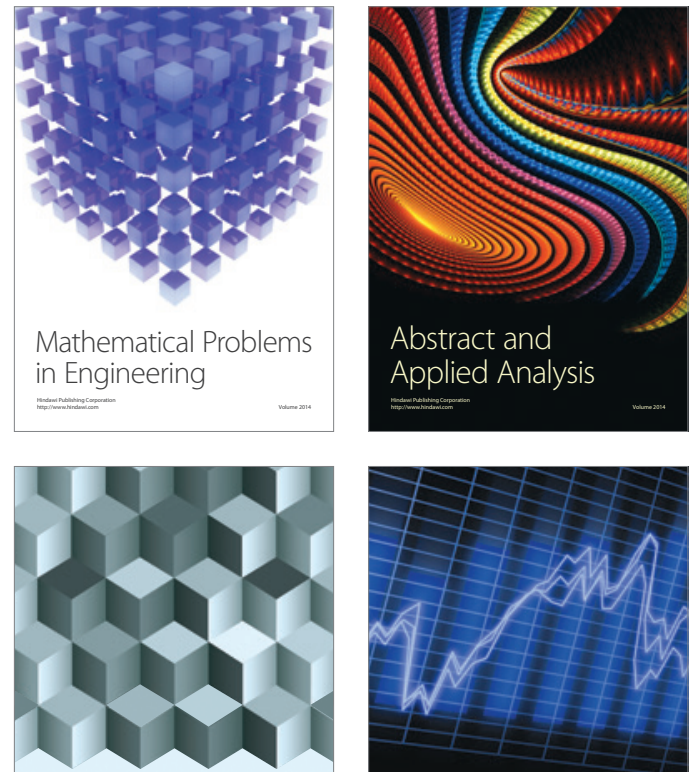

Journal of

Function Spaces

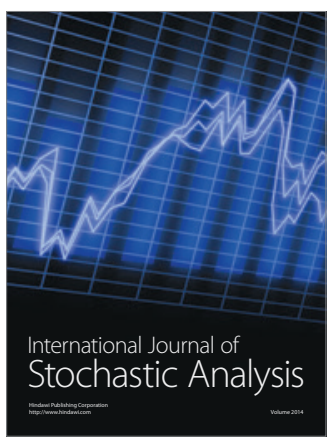

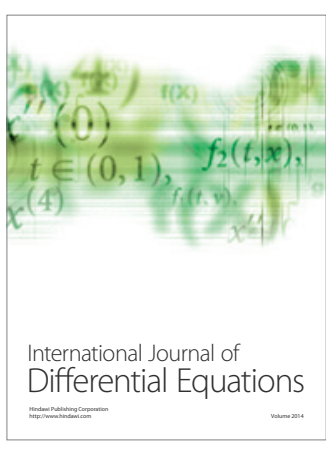
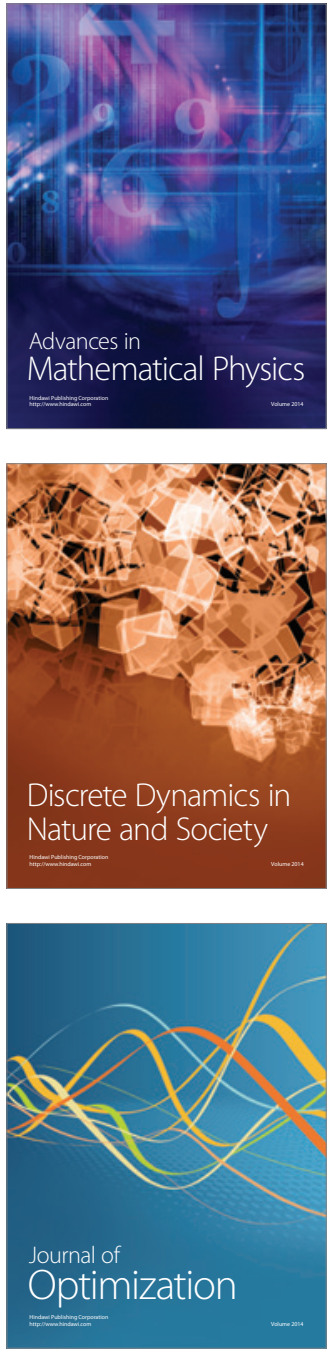